\title{
Truthful Mechanism Design for Wireless Powered Network With Channel Gain Reporting
}

\author{
Zhe Wang ${ }^{\circledR}$, Member, IEEE, Tansu Alpcan ${ }^{\circledR}$, Senior Member, IEEE, Jamie S. Evans ${ }^{\circledR}$, Senior Member, IEEE, \\ and Subhrakanti Dey ${ }^{\circledR}$, Senior Member, IEEE
}

\begin{abstract}
Directional wireless power transfer (WPT) technology provides a promising energy solution to remotely recharge the Internet of things sensors using directional antennas. Under a harvest-then-transmit protocol, the access point can adaptively allocate the transmit power among multiple energy directions to maximize the social welfare of the sensors, i.e., downlink sum received energy or uplink sum rate, based on full or quantized channel gains reported from the sensors. However, such power allocation can be challenged if each sensor belongs to a different agent and works in a competitive way. In order to maximize their own utilities, the sensors have the incentives to falsely report their channel gains, which unfortunately reduces the social welfare. To tackle this problem, we design the strategy-proof mechanisms to ensure that each sensor's dominant strategy is to truthfully reveal its channel gain regardless of other sensors' strategies. Under the benchmark full channel gain reporting (CGR) scheme, we adopt the Vickrey-Clarke-Groves (VCG) mechanism to derive the price functions for both downlink and uplink, where the truthfulness is guaranteed by asking each sensor to pay the social welfare loss of all other sensors attributable to its presence. For the 1-bit CGR scheme, the problem is more challenging due to the severe information asymmetry, where each sensor has true valuation of full channel gain but may report the false information of quantized channel gain. We prove that the classic VCG mechanism is no longer truthful and then propose two threshold-based price functions for both downlink and uplink, where the truthfulness is ensured by letting each sensor pay its own achievable utility improvement due to its participation. The numerical results validate the truthfulness of the proposed mechanism designs.
\end{abstract}

Index Terms-Directional wireless power transfer, limited channel feedback, game theory, mechanism design, dominantstrategy incentive-compatibility.

\section{INTRODUCTION}

A $S$ INTERNET of things (IoT) [1] is attracting more attentions nowadays, the application of wireless sensor network is of great research interest. Wireless power

Manuscript received November 25, 2018; revised April 15, 2019 and June 20, 2019; accepted July 24, 2019. Date of publication August 2, 2019; date of current version November 19, 2019. This work was supported in part by ARC Discovery under Project DP140101050. The associate editor coordinating the review of this article and approving it for publication was I. Krikidis. (Corresponding author: Zhe Wang.)

Z. Wang was with the Department of Electrical and Electronic Engineering, The University of Melbourne, Melbourne, VIC 3010, Australia. She is now with the Pillar of Engineering Systems and Design, Singapore University of Technology and Design, Singapore 487372 (e-mail: z.wang@sutd.edu.sg).

T. Alpcan and J. S. Evans are with the Department of Electrical and Electronic Engineering, The University of Melbourne, Melbourne, VIC 3010, Australia (e-mail: tansu.alpcan@unimelb.edu.au; jse@unimelb.edu.au).

S. Dey is with the Hamilton Institute, National University of Ireland Maynooth, Maynooth, W23 F2H6 Ireland (e-mail: Subhra.Dey@mu.ie).

Color versions of one or more of the figures in this article are available online at http://ieeexplore.ieee.org.

Digital Object Identifier 10.1109/TCOMM.2019.2932986 transfer (WPT) technology provides an alternative solution to prolong the lifetime of energy-limited wireless devices [2], where the energy transmitter, e.g., hybrid access points (APs) or power beacons, can recharge the remote wireless sensor nodes ( $\mathrm{SNs}$ ) via electromagnetic waves.

Since the RF signals decay quickly over distance, it is not energy efficient to omni-directionally broadcast energy in all directions. Directional WPT can increase the energy transfer efficiency by concentrating the RF energy to the directions of one or more sensors via energy beamforming [2]-[5], with the aid of multiple antennas or directional antennas. To achieve efficient power allocation, it is essential for the AP to know the channel power gain of each SN. The AP can obtain channel gain via channel training, i.e., each SN evaluates the channel gain from the pilot signal sent by the AP and then it reports the channel gain to the energy transmitter on the reverse link [6]-[9]. To reduce the feedback load and energy consumption, the SNs can feed the channel gain into a quantizer and return only a small number of bits to the transmitter, e.g., 1-bit feedback [11], [12]. One basic assumption of the aforementioned literature is that the SNs honestly follow the prescribed feedback protocols to report their true valuations of the channel gain to the AP. This works well for a single operator system but can be challenged in a self-interested system. For future IoT systems, the intelligent SNs may belong to different service providers and work in a selfish and competitive manner. In order to optimize its own utility, the SN may have the incentive to falsely report its private channel gain to the AP. This false-reporting behavior, in return, can deviate the resource allocation from the desired optimal outcome and reduce the social welfare of the SNs.

We adopt mechanism design theory to offset the falsereporting incentives of the SNs. Mechanism design [13], also known as reverse game theory, designs the payoff structure (i.e., price functions) to map the individual strategies of the rational players to the desired global outcome [14]. A mechanism is truthful (also named as incentive compatible) if each player maximizes its utility by disclosing its true private type (e.g., reporting the true valuation of channel gain to the AP) under such payoff structure. The stronger degree of truthful mechanism is dominant-strategy incentive compatibility (also called strategy-proofness), where truthtelling is a weakly dominant strategy for each player that earns a payoff no worse than any other strategies, regardless of the other SNs' strategies. The equilibrium in such a game is called a dominant-strategy equilibrium. In game theory, there 
is another term named Nash equilibrium is defined as the set of strategies for all the players such that no player can increase its utility (or payoff) by changing its strategy while others are playing according to their Nash equilibrium strategies. The most important dominant-strategy truthful mechanism is the Vickrey-Clarke-Groves (VCG) mechanism by Vickrey [15], Clarke [16], and Groves [17]. Holmstrom (1979) shows that, if the type space is smoothly connected, the VCG mechanism is the only direct mechanism that achieve both efficiency and strategy-proofness [18]. The VCG mechanism has been recently applied in wireless communications networks (e.g., ad hoc routing [19], wireless caching [20], spectrum trading [21] networks) to ensure that the agents truthfully reveal their private valuations, where the network resources (e.g., spectrum bandwidth) are treated as the divisible private good. For WPT system, the RF energy in each energy direction is a nonexclusive non-rival public good, which is different from the traditional applications.

\section{A. Contribution of This Work}

In this work, we focus on designing the mechanisms in a directional WPT network that achieves both efficiency and dominant-strategy truthfulness. We consider a harvest-thentransmit protocol, where the AP directionally transfers RF energy to the self-interested SNs in the downlink based on their reported channel gains, and then the SNs use the harvested energy to perform sensing tasks or transmit the information back to the AP in the uplink. We consider two channel gain reporting (CGR) schemes: full CGR and 1-bit CGR, where the SNs feed back the full or binary channel gains to the $\mathrm{AP}$, respectively. The AP, as the mechanism designer, aims to achieve the optimal power allocation that maximizes the social welfare of the SNs (i.e., the downlink sum received energy or uplink sum rate) based on the reported channel gains. The SNs, as the game players, target at finding the optimal strategies (i.e., reported channel gains) to maximize their own utilities under the power allocation and payoff structure (i.e., price function) designed by the AP. Since the SN and AP may have conflicting objectives, we need to design the truthful mechanism to align the local interests of the SNs with the global objective of the AP so that the truthful CGR is the dominant strategy of each SN and the corresponding social welfare is maximized. Moreover, we also have to guarantee each $\mathrm{SN}$ is individual rational, where no SN has negative utility and no SN gets worse off by participating in the game.

We face two major challenges in the mechanism design for directional WPT system. The first challenge is that, due to the multicast nature of directional WPT, the RF energy is not only a public good but also a private good. On the one hand, the RF energy within the same energy beam direction is a non-exclusive and non-rival public good, which means we cannot exclude one $\mathrm{SN}$ from free-riding on the wireless energy that is initially transmitted to another SN within the same energy direction. From this perspective, each SN may have the incentive to under-report its channel gain in order to free-ride on other SNs, i.e., to receive free energy without paying the proper price. On the other hand, the RF energy across different energy beam directions is an exclusive and rival private good, where increasing the energy allocated to one sector will reduce that allocated to other sectors. From this point of view, each SN may have the incentive to overreport its channel gain in order to win more energy over the competing SNs that are inside different energy directions. The dual property of the RF energy makes the mechanism design challenging. The second challenge is that the information is asymmetric between the AP and the SNs. The SNs have the exact knowledge of their private channel gains while the AP only receives the reported channel gain or even the quantized channel gains that partially reflect the private information. The VCG mechanism can be applied to the full CGR case as the type space is continuous. However, the mechanism design with 1-bit CGR is challenging due to the discrete type space and the classical VCG mechanism may not work.

We summarize our key contributions as follows.

- Optimal power allocation for the social welfare maximization: Under both CGR schemes, we derive the optimal power allocation for the directional WPT system to maximize the social welfare of all SNs, i.e., downlink sum received energy or uplink sum rate. Without any price function, we show that the SNs are not truthful in the Nash equilibrium under such power allocation, which reduces the corresponding social welfare.

- Dominant-strategy truthful mechanism with full CGR: For full CGR scheme, we adopt the VCG mechanism to design the price functions to ensure that truthful reporting is the dominant strategy for each SN. The truthfulness is guaranteed by asking each SN to pay the externality, i.e., the social welfare loss of all other SNs due to its participation. For the downlink truthful mechanism, each $\mathrm{SN}$ pays the amount of price that equals the reduction of the sum received energy of all other SNs. For the uplink truthful mechanism, an SN pays a non-zero price if it is the critical SN that reduces the water-level of the waterfilling power allocation, where the price equals the sum rate reduction of all other SNs.

- Dominant-strategy truthful mechanism with 1-bit CGR: For 1-bit CGR scheme, we prove that the VCG mechanism is not truthful, where the SNs can still improve their utilities by falsely reporting the quantized channel gain. To tackle this problem, we propose two novel threshold-based price functions for the downlink and uplink mechanisms, respectively, to ensure that each SN's dominant strategy is to truly reveal whether its channel gain is above a certain threshold. The truthfulness is guaranteed by asking each SN to pay the internality, i.e., the improvement of its own achievable utility attributable to its presence. For the downlink truthful mechanism, each SN pays the amount of price that equals the extra achievable energy received by itself. For the uplink truthful mechanism, each SN pays the amount of price that equals the achievable rate received by itself.

To our best knowledge, this work is the first attempt on the dominant-strategy truthful mechanism design for the directional WPT system. 


\section{B. Related Work}

Some recent work in [22]-[27] analyze the strategic interactions among selfish and competitive decision makers in wireless powered networks from a game-theoretic view. In [22], Nash equilibrium is considered as the solution of the bidding strategies in an energy auction game, where the nodes competitively bid for the RF energy from the energy transmitter and each pays a price proportional to its bid. [23] and [24] study two energy trading games, where [23] considers a Stackelberg game that one data AP purchases RF energy from several energy transmitters to recharge its sensor device and [24] considers an ascending-bid auction that multiple data APs purchase energy from one energy transmitter [24]. The work [25] extends [23] by considering imperfect channel information between the energy transmitter and the sensors is available at the data AP. However, [22]-[25] have the assumption that each node truthfully discloses its private information (e.g., the energy costs/requests or channel gains) to the resource allocator. Without this assumption, the equilibrium obtained in these schemes may deviate from the social optimality. The work [26] proposes an indirect Nash mechanism in the omnidirectional WPT scenario, where the users truthfully reveal their marginal utility (not the direct utility functions) to the energy transmitter when the decisions of all users converge to the Nash equilibrium. In [27], contract theory is adopted to establish the optimal contract between an data AP and several energy transmitters to maximize the data AP's expected throughput under the incentive compatible constraints at the energy transmitters.

Our work differs from the aforementioned literature in the following points. First, different from the indirect mechanisms adopted in [26] and [27], our work adopts a direct mechanism to derive the closed-form price function that guarantees truthful-reporting is the dominant strategy of each player in every realization regardless of other players' strategies, where dominant-strategy incentive-compatibility is the most desirable and strongest concept of truthful mechanism. Second, in our directional WPT framework, the RF energy is not only a public good but also a private good, where the RF energy is treated as a public good in [26] and as a private good in [22]-[25], [27]. Both the free-riding and competing incentives of the SNs make our mechanism design problem more challenging. Third, to save the feedback load and energy consumption, we consider a practical 1-bit CGR scheme that has not been addressed in any of the previous WPT mechanism design works. The false-reporting of the quantized channel gain causes a more severe information asymmetry problem, where the truthful mechanism design with limited private information is an open problem itself.

The rest of the paper is organized as follows. In Section II, we introduce the system model of the directional WPT with full CGR and 1-bit CGR. In Section III and Section IV, we design the dominant-strategy truthful mechanisms under the two CGR schemes for both downlink and uplink. Numerical results are shown and discussed in Section $\mathrm{V}$ to validate the truthfulness of the mechanisms. Finally, we draw conclusions in Section VI.

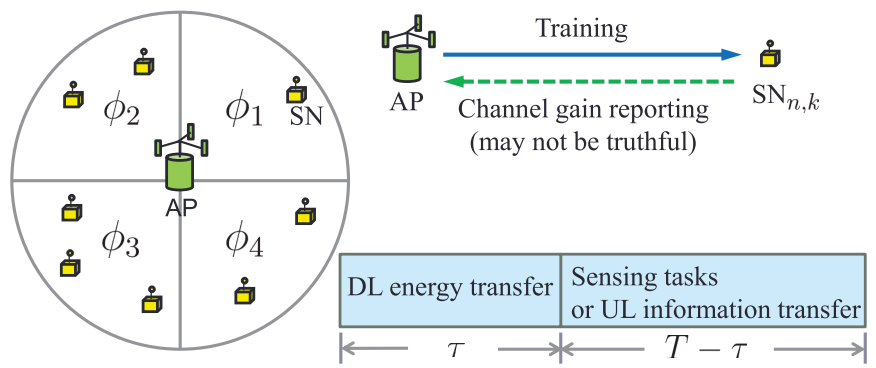

Fig. 1. System model for directional WPT with CGR. Based on the channel gains reported from the self-interested SNs, the AP adaptively allocates the RF energy among the multiple sectors in the downlink during $\tau$, and then the SNs perform sensing tasks or transmit information back to the AP in the uplink during $T-\tau$. The example is given for $N=4$.

\section{SYSTEM MODEL}

\section{A. Wireless Powered System With Channel Gain Reporting}

We consider a wireless powered network consisting of an $\mathrm{AP}$ and $L \mathrm{SNs}$, where the AP adaptively recharges the SNs inside its cell using directional antennas based on the reported channel gains from the SNs. As the directional WPT can significantly improve the energy transfer efficiency compared with the omnidirectional WPT, we consider a similar directional WPT scenario to our previous work in [4], where the AP is equipped with directional antennas (e.g., horn antennas) that can form energy beams in multiple directions. We divide the circular cell equally into $N$ non-overlapping sectors $\phi_{n}$ ( $n \in \mathbb{Z}_{N}=\{1,2 \cdots, N\}$ ) and the AP located at the cell center is able to form an energy beam in the direction of each intended sector. ${ }^{1}$ Different from [4], the AP does not simply allocate power equally to all intended sectors but adjusts the power intensity based on the reported channel gains from the SNs. A frame-based protocol is shown in Fig. 1. The AP first obtains channel gains of the SNs via channel training and feedback. And then we adopt the harvest-and-then transmit protocol for energy/information transfer.

We denote $K_{n}$ as the number of SNs fall inside the sector $\phi_{n}$ in a time frame and denote the SNs that are within this sector by $\mathrm{SN}_{n, k}\left(k \in \mathbb{Z}_{K_{n}}=\left\{1,2, \cdots, K_{n}\right\}\right)$. We may consider the SNs follow a Binomial point process, where a fixed number of $L \mathrm{SNs}$ are located randomly and independently inside the cell. In this setup, the users' locations are independent and identically distributed (i.i.d.) so that the resource allocation in later sections is fair (i.e., each sensor is equally likely to be served) from a long-term perspective. The main results and analysis do not depend on any specific point process. We assume the locations of the SNs are static within each time frame and can be different across the time frames. We denote the channel power gains between the AP and $\mathrm{SN}_{n, k}$ by $h_{n, k}$ which includes both large-scale fading (due to path loss and shadowing) and small-scale fading (due to reflection and diffraction). The small-scale fading channels are assumed to be reciprocal in the uplink and downlink and follow i.i.d.

\footnotetext{
${ }^{1}$ For the ease of analysis, we ignore the side lobes and assume the radiated energy is uniformly distributed across the main lobe of each energy beam [4] and assume the sectorization is ideal.
} 
quasi-static flat fading, which are constants within each time frame.

For efficient power allocation, it is important for the AP to know the instantaneous channel information via channel training and feedback [9], [28]. At the beginning of each time frame, the AP broadcasts a dedicated training sequence (known to the SNs) with equal power allocation among the sectors. By receiving the training sequence, each $\mathrm{SN}_{n, k}$ estimates the channel gain $h_{n, k}$. We consider the coherence interval and time frame are large enough so that the training process takes a negligible fraction of the total time in each block and essentially perfect channel state estimates can be obtained at the SNs [6], [10]. One can also adopt a fixed time duration for this feedback process, which will not change the main results on the mechanism design. After the channel estimation, the SNs report the full or 1-bit quantized channel gain to the AP through orthogonal and error-free feedback channels. ${ }^{2}$ In this work, we consider that the SNs may belong to different service providers, where each self-interested $\mathbf{S N}_{n, k}$ may report a different channel gain from its true valuation.

1) Full CGR: First, we consider the benchmark scenario that the SNs send full knowledge of channel gains to the $\mathrm{AP}$, where the reported channel gain for $\mathrm{SN}_{n, k}$ is denoted as $\tilde{h}_{n, k}$. For tractable analysis, we assume $h_{\max }$ (with sufficiently large value) as an upper bound for both the channel gain and reported channel gain throughout the paper. We define $\tilde{h}_{n, k}=h_{n, k}, \tilde{h}_{n, k}<h_{n, k}$ and $\tilde{h}_{n, k}>h_{n, k}$ as truthful reporting, under-reporting and over-reporting, respectively.

2) 1-Bit CGR: Second, we consider a more practical scenario where the SNs only report the quantized channel gain, i.e., 1-bit channel gain, to reduce the energy and bandwidth consumption. The channel gain is quantized into two regions, i.e., $[0, \gamma)$ and $\left[\gamma, h_{\max }\right)$, where $\gamma$ is the channel quantization threshold. After the channel training, each $\mathbf{S N}_{n, k}$ determines the true valuation of the binary channel indicator $b_{n, k}$ that is expressed as

$$
b_{n, k}= \begin{cases}0, & \text { if } h_{n, k} \in[0, \gamma) \\ 1, & \text { if } h_{n, k} \in\left[\gamma, h_{\max }\right) .\end{cases}
$$

And then $\mathrm{SN}_{n, k}$ reports the binary indicator $\tilde{b}_{n, k}$ to the AP, where " $\tilde{b}_{n, k}=0$ " or " $\tilde{b}_{n, k}=1$ " indicates whether its channel gain is below or above the threshold, respectively. ${ }^{3}$ If $\tilde{b}_{n, k}=0$ while its true valuation is $b_{n, k}=1$, we say $\mathrm{SN}_{n, k}$ is underreporting. Similarly, we say $\mathrm{SN}_{n, k}$ over-reports if $\tilde{b}_{n, k}=1$ while its true valuation is $b_{n, k}=0$.

After receiving the reported channel gain $\tilde{h}_{n, k}$ or $\tilde{b}_{n, k}$ from the SNs, we adopt a harvest-then-sense/transmit protocol for the energy/information transfer processes. Each time frame

\footnotetext{
${ }^{2}$ We assume the energy used for channel reporting is small, e.g., the energy used for 1-bit feedback is negligible [11], which can be either supported by a dedicated battery or recharged by the AP periodically with short broadcast pulses. Furthermore, we assume the channel gain reporting is error-free [11], which can be well approximated via the use of sufficiently powerful error control codes over the feedback link [29]. It would be also interesting to investigate the measurement errors in the channel reporting process, which however, are beyond the scope of this paper. The focus of this work is to prevent the subjective false-reporting incentives of SNs.

${ }^{3}$ From the long-term energy saving perspective, we may also let the SNs be silent if $\tilde{b}_{n, k}=0$.
}

$T$ is divided into two phases: energy transfer phase $\tau$ and sensing/transmit phase $T-\tau$ [3], [30]. During $\tau$, the AP directionally transmits wireless energy to the SNs based on the reported channel gains and each SN temporarily stores the harvested energy in a capacitor. ${ }^{4}$ During $T-\tau$, the SNs use the harvested energy to perform sensing tasks or to report the information back to the AP with best effort. Since capacitor can be charged and discharged quickly and can only store energy for a short period of time, we consider the energy in the capacitor is used up within the current time frame and cannot be carried forward to the next frame [4]. In Sections III and IV, we will discuss the optimal power allocation to each sector/SN according to the different social welfare maximization objectives and the corresponding price function designs that ensure the truthfulness of channel reporting.

\section{B. Game Components and Mechanism Design}

We formulate the game components as follows. The SNs are the players each has a private channel gain $h_{n, k}$ that is hidden from the AP. The strategy of each $\mathrm{SN}_{n, k}$ is to report the channel gain to the AP, i.e., $\tilde{h}_{n, k}$ for full CGR or $\tilde{b}_{n, k}$ for 1-bit CGR, under different reporting schemes. We assume all self-interested SNs are non-cooperative and rational with the aim of maximizing its own utility, i.e., downlink received energy or uplink rate. Based on the reported channel gains, the AP with total transmit power $P_{t o t}$ allocates power $P_{n}$ to the direction of each sector $\phi_{n}$ to maximize the social welfare, i.e., sum received energy or sum rate of all SNs. ${ }^{5}$ Since the SNs are selfish, they may not report their true valuations of the channel gains to the AP. In Sections III and IV, we will show that the SNs have the incentives to dishonestly report their channel gains, which deviates the power allocation of the AP from the optimal solution and thus reduces the social welfare. To offset this false-reporting incentive, the AP therefore has to charge a price $C_{n, k}$ from each $\mathrm{SN}_{n, k}$ for providing the resources of energy or time. We consider the AP as a non-profit public service provider which aims at efficiently allocating the resources to maximize the social welfare of the SNs instead of maximizing its own revenue. To ensure that each user truthfully reveals its private channel gain, the AP charges a certain price from each SN in forms of virtual credit [19], [20]. We consider the SNs are price takers and they can only affect their payment via the reported channel gains.

To give a brief picture first, we describe the interaction between the $\mathrm{AP}$ and SNs in Fig. 2. Each $\mathrm{SN}_{n, k}$ targets at finding the best reporting strategy $\tilde{h}_{n, k}$ (or $\tilde{b}_{n, k}$ ) that maximizes its own utility $U_{n, k}\left(h_{n, k}, P_{n}, C_{n, k}\right)$ under the power allocation $P_{n}$ and price function $C_{n, k}$ designed by the AP. The $\mathrm{AP}$, as the mechanism designer has two goals: (1) to obtain the power allocation $P_{n}$ for each sector $\phi_{n}$ that maximizes the social welfare, i.e., sum received energy or sum rate of all SNs; (2) to design the price function $C_{n, k}$ that ensures the

\footnotetext{
${ }^{4} \mathrm{We}$ assume the energy storage of the capacitors is sufficiently large (e.g., supercapacitors), where the SNs can use as much power as it receives in a time frame.

${ }^{5}$ Since the channel power gains (including both large-scale and small-scale fading) between the AP and users are i.i.d, each user are equally likely to be served from an average perspective and the scheme is fair in a long run.
} 


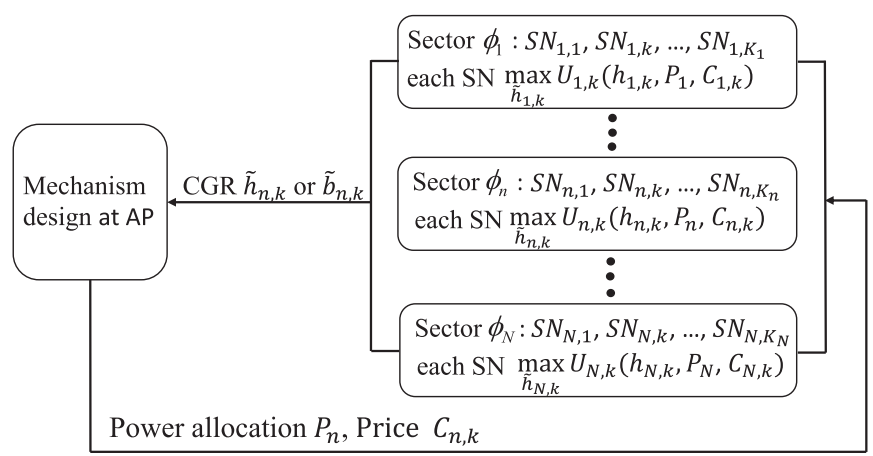

Fig. 2. System diagram of mechanism design for directional WPT.

truthful reporting (i.e., $\tilde{h}_{n, k}=h_{n, k}$ or $\tilde{b}_{n, k}=b_{n, k}$ ) is the dominant strategy for each $\mathrm{SN}_{n, k}$.

\section{TRuthful Mechanism Design For Downlink SUM ENERGY MAXIMIZATION}

In this section, we consider the scenario that the SNs harvest energy from the AP during $\tau$ and then use up the harvested energy to perform the sensing tasks during $T-\tau$ with best effort. We design the dominant-strategy truthful mechanism for the full CGR and 1-bit CGR schemes under the optimal power allocation for the downlink sum energy maximization. We first derive the optimal power allocation of the AP to maximize the downlink sum received energy at the SNs. Then, we show that the SNs are not truthful at the Nash equilibrium under such power allocation. Finally, we design the price functions to ensure that truthful CGR is the dominant strategy for each SN.

In the downlink energy transfer slot $\tau$, the AP allocates the amount of power $P_{n}\left(P_{n} \geq 0\right)$ to the direction of the sector $\phi_{n}(n=1,2, \cdots, N)$ out of the total transmit power $P_{t o t}$ based on the reported channel gains. By receiving $\tilde{h}_{n, k}$ or $\tilde{b}_{n, k}$ from each $\mathrm{SN}_{n, k}$, the $\mathrm{AP}$ evaluates the received energy $W_{n, k}\left(\tilde{h}_{n, k}, P_{n}\right)$ or achievable received energy ${ }^{6} W_{n, k}\left(\tilde{b}_{n, k} \gamma, P_{n}\right)$ at $\mathbf{S N}_{n, k}$, i.e.,

$$
W_{n, k}\left(\tilde{h}_{n, k}, P_{n}\right)= \begin{cases}\tau \delta \tilde{h}_{n, k} P_{n}, & \text { for full CGR } \\ \tau \delta \tilde{b}_{n, k} \gamma P_{n}, & \text { for 1-bit CGR, }\end{cases}
$$

where $\delta$ is the product of the directional antenna gain and the energy conversion efficiency. ${ }^{7}$ To obtain tractable results, we adopt the linear energy harvesting model [2], [31], where the harvested DC power at the $\mathrm{SN}$ is proportional to the input RF power. For low received power regime (e.g., less than $10 \mathrm{~mW}$ ), the linear energy harvesting model gives a good approximation to the more practical non-linear model [32]. We denote the sum of SNs' reported channel gains in the sector $\phi_{n}$ with full CGR by $\alpha_{n}=\sum_{k \in \mathbb{Z}_{K_{n}}} \tilde{h}_{n, k}$ and that with 1-bit CGR by $\beta_{n}=\sum_{k \in \mathbb{Z}_{K_{n}}} \tilde{b}_{n, k}$. From the AP's view, the sum of received energy/achievable received energy at all SNs with

\footnotetext{
${ }^{6}$ Throughout the paper, we use the term "achievable" to describe the effective energy/rate of the SNs from the AP's perspective for 1-bit CGR. Here, the channel gain is taken as $\gamma$ when $\tilde{b}_{n, k}=1$ or 0 when $\tilde{b}_{n, k}=0$, respectively.

${ }^{7} \mathrm{We}$ assume the noise power at each $\mathrm{SN}$ is not taken into account for the harvested energy.
}

full CGR/1-bit CGR is given by

$$
W_{\text {sum }}\left(P_{n}\right)= \begin{cases}\tau \delta \sum_{n \in \mathbb{Z}_{N}} P_{n} \alpha_{n}, & \text { for full CGR } \\ \tau \delta \gamma \sum_{n \in \mathbb{Z}_{N}} P_{n} \beta_{n}, & \text { for 1-bit CGR. }\end{cases}
$$

\section{A. Optimal Power Allocation for Downlink Sum Energy Maximization}

In this section, we consider the scenario that the SNs use up the harvested energy during $\tau$ to perform the sensing tasks during $T-\tau$ with best effort. In P1 defined below, the AP aims to optimally allocate power to each sector so that the sum received energy for all SNs is maximized under the total transmit power constraint $P_{t o t}$.

$$
\begin{aligned}
\text { P1 : } \max _{P_{n}} & W_{\text {sum }}\left(P_{n}\right) \\
\text { s.t. } & \sum_{n \in \mathbb{Z}_{N}} P_{n} \leq P_{\text {tot }} \\
& P_{n} \geq 0 .
\end{aligned}
$$

$\mathrm{P} 1$ is a linear programming problem, where the solution is at the boundary of the feasible region. Here we consider the AP has directional antenna, where a similar problem with MIMO setup was solved in [2]. In P1, we arrange the sum of the reported channel gain in each sector in ascending order and denote the highest value as $\alpha_{(N)}=\max _{n \in \mathbb{Z}_{N}}\left\{\alpha_{n}\right\}$ for the full CGR and $\beta_{(N)}=\max _{n \in \mathbb{Z}_{N}}\left\{\beta_{n}\right\}$ for the 1-bit CGR. We include the possibility of ties by considering that the sum of reported CGR for different sectors can be the same (e.g., we may have $\alpha_{i}=\alpha_{j}$ for $i \neq j$ ). We consider the AP equally allocates the power to all sectors in a tie. Basically, using a different tie-breaking rule (e.g., either chooses one user or a subset of users as the winners) will not change the main conclusion about the truthfulness as long as the players are indifferent and the winner is randomly selected. By solving $\mathrm{P} 1$, the optimal power allocation in the direction of sector $\phi_{n}$ is given by

$$
P_{n}^{*}= \begin{cases}\frac{P_{t o t}}{M}, & \text { if } \alpha_{n}=\alpha_{(N)} \text { for full CGR } \\ 0, & \text { or } \beta_{n}=\beta_{(N)} \text { for 1-bit CGR } \\ 0 \text { if } \alpha_{n}<\alpha_{(N)} \text { for full CGR } \\ & \text { or } \beta_{n}<\beta_{(N)} \text { for 1-bit CGR, }\end{cases}
$$

where $M \geq 1$ is the number of sectors with the sum of reported channel gain equals $\alpha_{(N)}$ (or $\beta_{(N)}$ ). In (7), the AP divides the total energy among those sectors that have the highest sum of reported channel gains.

\section{B. Nash Equilibrium and Downlink Price Function}

In this subsection, we first discuss the Nash equilibrium of the self-interested SNs when no price is paid to the AP for energy receiving. By showing the SNs have untruthful reporting incentives, we then introduce the price function and the design criteria of the dominant-strategy truthful mechanism.

As $\mathrm{SN}_{n, k}$ has the knowledge of true channel gain $h_{n, k}$, its true valuation of received energy is

$W_{n, k}\left(h_{n, k}, P_{n}\right)=\tau \delta h_{n, k} P_{n}$, for $n \in \mathbb{Z}_{n}$ and $k \in \mathbb{Z}_{K_{n}}$, 
Under the AP's power allocation in (7), each self-interested $\mathrm{SN}_{n, k}$ chooses its optimal reporting strategy to maximize its received energy. For full CGR, we have

$$
\begin{aligned}
\text { P2 : } & \max _{\tilde{h}_{n, k}} W_{n, k}\left(h_{n, k}, P_{n}^{*}\left(\tilde{h}_{n, k}\right)\right) \\
& \text { s.t. } \tilde{h}_{n, k} \in\left[0, h_{\max }\right],
\end{aligned}
$$

and for 1-bit CGR we replace $\tilde{h}_{n, k}$ by $\tilde{b}_{n, k} \gamma$ in P2, where $\tilde{b}_{n, k} \in\{0,1\}$. Based on (7), $P_{n}^{*}$ is non-decreasing with the increase of $\tilde{h}_{n, k}$ or $\tilde{b}_{n, k}$. In order to increase the received energy, each $\mathrm{SN}_{n, k}$ has the incentive to over-report. By solving $\mathrm{P} 2$, the Nash equilibrium with full CGR is $\tilde{h}_{n, k}^{*}=h_{\max }$ and that with 1-bit CGR is $\tilde{b}_{n, k}^{*}=1 \forall n, k$. However, this Nash equilibrium is not efficient. One can easily check that the false-reporting in the Nash equilibrium can lead to substantial decrease of the sum received energy compared with that of the truthful reporting.

To offset the over-reporting incentives of the SNs, the AP charges a price $C_{n, k}$ from each $\mathrm{SN}_{n, k}$ as a cost for receiving energy. For each $\mathrm{SN}_{n, k}$, the utility function in the downlink is defined as

$$
U_{n, k}= \begin{cases}W_{n, k}\left(h_{n, k}, P_{n}^{*}\left(\tilde{h}_{n, k}\right)\right)-C_{n, k}, & \text { for full CGR } \\ W_{n, k}\left(h_{n, k}, P_{n}^{*}\left(\tilde{b}_{n, k}\right)\right)-C_{n, k}, & \text { for 1-bit CGR. }\end{cases}
$$

With the price function $C_{n, k}$, the optimization problem in P2 becomes

$$
\begin{aligned}
\text { P3 : } & \max _{\tilde{h}_{n, k}} U_{n, k}\left(h_{n, k}, P_{n}^{*}\left(\tilde{h}_{n, k}\right)\right) \\
& \text { s.t. } \tilde{h}_{n, k} \in\left[0, h_{\max }\right]
\end{aligned}
$$

for full CGR. And for 1-bit CGR we replace $\tilde{h}_{n, k}$ by $\tilde{b}_{n, k} \gamma$ in $\mathrm{P} 3$, where $\tilde{b}_{n, k} \in\{0,1\}$. In the following two subsections, we will design the price function $C_{n, k}$ for each SN to ensure that truthful reporting (i.e., $\tilde{h}_{n, k}=h_{n, k}$ for full CGR and $\tilde{b}_{n, k}=b_{n, k}$ for 1-bit CGR) is the dominant strategy for each $\mathrm{SN}$ in $\mathrm{P} 3$.

\section{Downlink Price Function Design With Full CGR}

In this subsection, we design the price function for downlink sum energy maximization with full CGR. Based on the power allocation in (7), only the sectors that have the highest sum of reported channel gains receive non-zero power allocation from the AP. A straightforward pricing scheme is the proportional pricing scheme. Similar to [22], we can let each SN pay a price that is proportional to its reported channel gain. However, due to the non-exclusive and non-rival property of the wireless energy, the SNs may have the incentive to under-report their channel gains under such pricing scheme in order to free ride on other users within the same sector.

In order to guarantee each SN truthfully reports its channel gain, we now adopt the VCG mechanism to derive the price functions. The key idea of the VCG mechanism is that each SN pays the "externality" it imposes on all other SNs due to its participation, where the "externality" is the change of the social welfare (e.g., sum received energy or sum rate) for the rest of the SNs in the system. For downlink energy transfer scheme, the participation of $\mathrm{SN}_{n, k}$ may improve the energy of the SNs that are also within sector $\phi_{n}$ and reduce that of the SNs in other sectors. By adopting the VCG mechanism, $\mathrm{SN}_{n, k}$ pays the difference between the sum received energy of all other SNs (both intra-sector and inter-sector) under the efficient power allocation due to its participation, i.e.,

$$
\begin{aligned}
& C_{n, k}=\sum_{\substack{i \in \mathbb{Z}_{N},(i, j) \neq(n, k)}} \sum_{j \in \mathbb{Z}_{K_{i}}} W_{i, j}\left(\tilde{h}_{i, j}, P_{i}^{* *}\right) \\
& -\sum_{\substack{i \in \mathbb{Z}_{N}, j \in \mathbb{Z}_{K_{i}} \\
(i, j) \neq(n, k)}} W_{i, j}\left(\tilde{h}_{i, j}, P_{i}^{*}\left(\tilde{h}_{n, k}\right)\right),
\end{aligned}
$$

where $P_{i}^{* *}$ and $P_{i}^{*}$ (given in (7)) are the optimal power allocations for the sector $\phi_{i}$ without and with the participation of $\mathrm{SN}_{n, k}$, respectively.

Theorem 1: For full CGR, the VCG price in (14) ensures that truthful reporting of $\tilde{h}_{n, k}=h_{n, k}$ is the dominant strategy for each $\mathrm{SN}_{n, k}$ in $\mathrm{P} 3$ under the power allocation in P1.

\section{Proof: See Appendix A.}

We now derive $P_{i}^{* *}$ in (14) as follows. Without the participation of $\mathrm{SN}_{n, k}$, the power allocation problem at the $\mathrm{AP}$ is given by

$$
\begin{array}{cl}
\text { P4: } \max _{P_{i}} W_{\text {sum }}-W_{n, k}=\sum_{\substack{i \in \mathbb{Z}_{N},(i, j) \neq(n, k)}} \sum_{j \in \mathbb{Z}_{K_{n}}} W_{i, j} \\
\text { s.t. } \sum_{i \in \mathbb{Z}_{N}} P_{i} \leq P_{\text {tot }} \\
P_{i} \geq 0 .
\end{array}
$$

We denote the sum of reported channel gains of the rest of the SNs in the sector $\phi_{n}$ as $\hat{\alpha}_{n}=\alpha_{n}-\tilde{h}_{n, k}$. We rearrange the sum of the reported channel gains $\alpha_{1}, \alpha_{2}, \cdots, \hat{\alpha}_{n}, \cdots, \alpha_{N}$ in ascending order and denote the new highest value among them as $\hat{\alpha}_{(N)}=\max _{i \in \mathbb{Z}_{N}}\left\{\alpha_{i \neq n}, \hat{\alpha}_{n}\right\}$. Similar to P1, the optimal power allocation for $\mathrm{P} 4$ is to equally allocate the total power $P_{\text {tot }}$ to the $\hat{M}$ sectors $(\hat{M} \geq 1)$ that each has the sum of the reported channel gains equals $\hat{\alpha}_{(N)}$, i.e.,

$$
P_{i}^{* *}= \begin{cases}\frac{P_{t o t}}{\hat{M}}, & \text { if } \alpha_{i}=\hat{\alpha}_{(N)} \text { for } i \neq n \\ & \text { or } \hat{\alpha}_{i}=\hat{\alpha}_{(N)} \text { for } i=n \\ 0, & \text { if } \alpha_{i}<\hat{\alpha}_{(N)} \text { for } i \neq n \\ & \text { or } \hat{\alpha}_{i}<\hat{\alpha}_{(N)} \text { for } i=n\end{cases}
$$

By substituting $P_{i}^{*}$ in (7) and $P_{i}^{* *}$ in (18) into $C_{n, k}$ in (14), the first and second terms of the right handside of (14) are given by

$$
\begin{aligned}
\sum_{\substack{i \in \mathbb{Z}_{N}, j \in \mathbb{Z}_{K_{i}} \\
(i, j) \neq(n, k)}} W_{i, j}\left(h_{i, j}, P_{i}^{* *}\right) & =\tau \delta P_{t o t} \hat{\alpha}_{(N)} \\
& = \begin{cases}\tau \delta P_{t o t} \alpha_{(N)}, & \text { if } \alpha_{n}<\alpha_{(N)} \\
\tau \delta P_{t o t} \hat{\alpha}_{(N)}, & \text { if } \alpha_{n}=\alpha_{(N)}\end{cases}
\end{aligned}
$$


and

$$
\begin{aligned}
& \sum_{\substack{i \in \mathbb{Z}_{N}, j \in \mathbb{Z}_{K_{i}} \\
(i, j) \neq(n, k)}} W_{i, j}\left(h_{i, j}, P_{i}^{*}\left(h_{n, k}\right)\right) \\
& = \begin{cases}\tau \delta P_{t o t} \alpha_{(N)}, & \text { if } \alpha_{n}<\alpha_{(N)} \\
\tau \delta P_{t o t}\left(\alpha_{(N)}-\frac{\tilde{h}_{n, k}}{M}\right), & \text { if } \alpha_{n}=\alpha_{(N)} \text { and } \hat{\alpha}_{n}<\hat{\alpha}_{(N)} \\
\tau \delta P_{t o t} \hat{\alpha}_{(N)}, & \text { if } \alpha_{n}=\alpha_{(N)} \text { and } \hat{\alpha}_{n}=\hat{\alpha}_{(N)} .\end{cases}
\end{aligned}
$$

From (19) and (20), we observe that the participation of $\mathrm{SN}_{n, k}$ changes the sum of received energy of all other SNs only if $\alpha_{n}=\alpha_{(N)}$ and $\hat{\alpha}_{n}<\hat{\alpha}_{(N)}$ hold at the same time, which means the sum of the reported channel gains in the sector $\phi_{n}$ is not the highest among all sectors unless $\mathrm{SN}_{n, k}$ participates. Substituting (19) and (20) into (14), the price function for $\mathrm{SN}_{n, k}$ is given in the following proposition.

Proposition 1: Under the optimal power allocation for downlink sum energy maximization in (7), the VCG price of

$$
C_{n, k}= \begin{cases}\tau \delta P_{t o t}\left[\hat{\alpha}_{(N)}-\alpha_{(N)}+\frac{\tilde{h}_{n, k}}{M}\right], & \text { if } \alpha_{n}=\alpha_{(N)} \\ 0, & \text { and } \hat{\alpha}_{n}<\hat{\alpha}_{(N)} \\ \text { otherwise. }\end{cases}
$$

ensures that truthful-reporting of the full CGR (i.e., $\tilde{h}_{n, k}=$ $h_{n, k}$ ) is the dominant strategy for each $\mathrm{SN}_{n, k}$ in P3. And each $\mathrm{SN}$ is individual rational under this VCG price.

Proof: See Appendix B.

Remark 1: Based on Proposition 1, if $\mathrm{SN}_{n, k}$ is the critical SN that changes the power allocation due to its participation, the price it pays equals to the change in the sum received energy of all other SNs, which includes both the increased energy of the other SNs within the sector $\phi_{n}$ and the reduced energy of SNs in all other sectors. Adopting the price in (21), each SN maximizes its own utility when it reports the true valuation of the channel gain to the AP. By receiving the truthful reported channel gains, the AP allocates the power according to (7) and the social welfare (i.e., sum received energy of all SNs) is thus maximized.

Remark 2: The price function, e.g. in (14) and (21) are computed by the mechanism designer. Although the function is complicated, it is computed in a transparent manner that is auditable, i.e. SNs can check these after the fact if necessary to ensure its fair application to all participants. In a practical implementation, SNs can agree to a contract clearly detailing the mechanism including after-the-fact audit options. This would give SNs motivation to abide by mechanism rules, which ensures efficiency and strategy-proofness for all participants.

\section{Downlink Price Function Design With 1-Bit CGR}

In this subsection, we discuss a more practical scenario of 1-bit CGR. After channel training, the SNs have the full information of their true channel valuation of $h_{n, k}$ but only feed back 1-bit channel gain $\tilde{b}_{n, k}$ to the AP. The design of the price function is more challenging due to the discrete type space $\left(\tilde{b}_{n, k} \in\{0,1\}\right)$, where it is difficult for the AP to offset the misreporting incentive based only on the partial reported channel gain.

1) Failure of VCG Mechanism: We first see if the VCG mechanism is also applicable to 1-bit CGR. In the absence of $\mathrm{SN}_{n, k}$, we denote the sum of the 1-bit CGR for the sector $\phi_{n}$ by $\hat{\beta}_{n}=\beta_{n}-\tilde{b}_{n, k}$. We denote $\hat{M} \geq 1$ as the number of sectors each with the sum of the CGR equals $\hat{\beta}_{(N)}=\max _{i \in \mathbb{Z}_{N}}\left\{\beta_{i \neq n}, \hat{\beta}_{n}\right\}$. For 1-bit CGR, we can obtain the VCG price similar to (21) and the utility function similar to $(42 \mathrm{a})-(42 \mathrm{c})$ by replacing $\alpha$ by $\beta$ and replacing $\tilde{h}_{n, k}$ by $\tilde{b}_{n, k} \gamma$. For $\beta_{n}=\beta_{(N)}$ and $\hat{\beta}_{n}<\hat{\beta}_{(N)}$, the sum of the reported channel gains in the sector $\phi_{n}$ is the highest with the participation of $\mathrm{SN}_{n, k}$ and is not the highest in the absence of $\mathrm{SN}_{n, k}$, which implies $\tilde{b}_{n, k}=1$. Since $\beta_{n}=\hat{\beta}_{n}+1$ and $\hat{\beta}_{(N)} \leq \beta_{(N)}$, we can deduce that $\hat{\beta}_{(N)}=\beta_{(N)}$. Under the VCG price, the utility function of $\mathrm{SN}_{n, k}$ with 1-bit CGR is given by

$$
U_{n, k}= \begin{cases}\tau \delta P_{t o t} h_{n, k} / M, & \text { if } \beta_{n}=\beta_{(N)} \\ \tau \delta P_{t o t}\left[h_{n, k} / M-\tilde{b}_{n, k} \gamma / M\right], & \text { and } \hat{\beta}_{n}=\hat{\beta}_{(N)} \\ & \text { if } \beta_{n}=\beta_{(N)} \\ 0, & \text { and } \hat{\beta}_{n}<\hat{\beta}_{(N)} \\ & \text { if } \beta_{n}<\beta_{(N)} .\end{cases}
$$

Counterexample of VCG Mechanism: Though truthful reporting of $\tilde{b}_{n, k}=b_{n, k}$ maximizes $U_{n, k}$ in the last two cases of (22), $\mathrm{SN}_{n, k}$ is able to increase its utility $U_{n, k}$ by overreporting in the first case. We now give an example to prove the untruthfulness of VCG for the first case of (22). Consider $\hat{M}>1$ and $0<h_{n, k}<\gamma$. By truthfully reporting with $\tilde{b}_{n, k}=0$, we have $M=\hat{M}>1$ and the utility of $\mathrm{SN}_{n, k}$ is $\tau \delta P_{\text {tot }} h_{n, k} / M$. By over-reporting with $\tilde{b}_{n, k}=1$, the sector $\phi_{n}$ becomes the only one sector (i.e., $M=1$ ) that has the highest value of sum of channel gains among all sectors and its utility is $\tau \delta P_{t o t} h_{n, k}$. In this case, $\mathrm{SN}_{n, k}$ has the incentive to cheat since it receives more energy by paying nothing based on the VCG price.

Remark 3: For 1-bit CGR, the AP designs the power allocation and price functions only based on the partial channel information which is already distorted from the true valuation due to quantization. The information is thus severely asymmetric between the AP and the SN. Each sensor has true valuation of full channel gain but may report the false information of quantized channel gain. Due to the gap between the achievable received energy deduced by the AP and the actual energy received at the SNs, the VCG price may sometimes not big enough to offset the false reporting incentives of the SNs. The SNs can take the advantage of this information asymmetry when choosing their best responses of $\tilde{b}_{n, k}$ to maximize their own utilities. In this case, the VCG mechanism does not always guarantee the truthfulness and there is still a room for some SNs to be dishonest.

2) Threshold-Based Price Function: For 1-bit CGR, we propose a price function based on the channel quantization threshold in the following theorem. 
Theorem 2: Under the optimal power allocation for downlink sum energy maximization, the threshold-based price function of

$$
C_{n, k}=\tau \delta \tilde{b}_{n, k} \gamma\left(P_{n}^{*}-P_{n}^{* *}\right)
$$

ensures that truthful-reporting of the 1-bit CGR (i.e., $\tilde{b}_{n, k}=$ $\left.b_{n, k}\right)$ is the dominant strategy for each $\mathbf{S N}_{n, k}$ in $\mathrm{P} 3$, where $P_{n}^{*}=\mathbb{1}\left(\beta_{n}=\beta_{(N)}\right) P_{t o t} / M$ and $P_{n}^{* *}=\mathbb{1}\left(\hat{\beta}_{n}=\right.$ $\left.\hat{\beta}_{(N)}\right) P_{t o t} / \hat{M}$ are the optimal power allocated to sector $\phi_{n}$ with and without the participation of $\mathrm{SN}_{n, k}$, respectively. And each $\mathrm{SN}$ is individual rational under this threshold-based price.

Proof: See Appendix C.

Remark 4: From Theorem 2, we see that $\mathrm{SN}_{n, k}$ pays a nonzero price if it is the critical SN that changes the optimal power allocation due to its participation. Different from the VCG price that depends on the loss of the sum received energy of all other SNs (except $\mathrm{SN}_{n, k}$ ), the threshold-based price depends on the extra achievable energy received by $\mathrm{SN}_{n, k}$. Since the RF energy on the direction of $\phi_{n}$ is a non-exclusive and nonrival public good, we need to subtracts $P_{n}^{* *}$ from $P_{n}^{*}$ in (23) in order to remove the free-riding incentive of $\mathrm{SN}_{n, k}$ on other users. By exploiting the unique property of the quantization threshold, (23) ensures that truthful reporting is the dominant strategy of each $\mathrm{SN}$.

Remark 5: To ensure individual rationality of each SN, the AP under-estimates the utilities of the SNs by using the lower boundaries of the channel quantization interval (i.e., 0 and $\gamma$ ) for power allocation and price designs. If the AP assumes the gains of the SNs according to the middles of the intervals, i.e., $\gamma / 2$ and $\left(\gamma+h_{\max }\right) / 2$, it may overestimate the welfare of the $\mathrm{SNs}$ which channel gains are within $[0, \gamma / 2]$ or $\left[\gamma,\left(\gamma+h_{\max }\right) / 2\right]$ and will thus charge them a high price accordingly. As such, these SNs may result in negative utility, which violates the requirement of individual rationality.

\section{Truthful Mechanism Design for Uplink Sum Rate Maximization}

In this section, we consider the scenario that the SNs harvest energy from the AP during $\tau$ and then use up the harvested energy to transmit information back to the AP during $T-\tau$ with best effort. We design the dominant-strategy truthful mechanism for full CGR and 1-bit CGR schemes under the optimal power allocation for the uplink sum rate maximization. For each CGR scheme, we first design the price functions to ensure that truthful CGR is the dominant strategy for each SN.

\section{A. Sum Rate in the Uplink}

1) Uplink Information Transfer With Full CGR: After channel training, each $\mathrm{SN}_{n, k}$ obtains $h_{n, k}$ in this fading block and reports the channel gain $\tilde{h}_{n, k}$ back to the AP to aid the downlink power allocation. By harvesting the energy of $W_{n, k}\left(h_{n, k}, P_{n}\left(\tilde{h}_{n, k}\right)\right)$ from the AP, each $\mathrm{SN}_{n, k}$ uses up this energy to transmit information to the AP in an orthogonal manner, e.g., TDMA. For full CGR, we divide the information transfer slot $T-\tau$ into $K_{n}$ sub-slots and allow each $\mathrm{SN}_{n, k}$ to transmit during a sub-slot of $(T-\tau) / K_{n}$ with power of
$W_{n, k}\left(h_{n, k}, P_{n}\left(\tilde{h}_{n, k}\right)\right) K_{n} /(T-\tau)$. We assume the additive white Gaussian noise at the AP has zero mean and variance $\sigma$. Since the AP may not fully trust on the reported channel gain $\tilde{h}_{n, k}$, it adopts non-coherent detection that does not depend on the knowledge of channel gain. On the other hand, as $\mathbf{S N}_{n, k}$ knows the true channel gain $h_{n, k}$, it transmits information with a maximum rate within its channel capacity. Then, the AP decodes the uplink information using the non-coherent detection techniques with sophisticated decoders that take the uplink rate close to the capacity bounds (e.g., see [33] and the reference therein). Throughout this section, we treat the uplink rate as an upper bound only, whereas investigating more sophisticated coding/decoding for characterizing the exact uplink sum rate is beyond the scope of the current paper, and is left for future work. With a slight abuse of notation, we denote the upper bound of uplink rate as uplink rate in the rest of this paper. From the perspective of SN, the uplink rate is

$$
\begin{aligned}
& R_{n, k}\left(h_{n, k}, P_{n}, K_{n}\right) \\
& \quad=\frac{T-\tau}{K_{n}} \log \left[1+\frac{h_{n, k} W_{n, k}\left(h_{n, k}, P_{n}\left(\tilde{h}_{n, k}\right)\right) K_{n}}{(T-\tau) \sigma}\right] .
\end{aligned}
$$

From the perspective of the AP, the uplink sum rate of all SNs is given by

$$
\begin{aligned}
& R_{\text {sum }}\left(\tilde{h}_{n, k}, P_{n}, K_{n}\right) \\
& \quad=\sum_{n \in \mathbb{Z}_{N}} \sum_{k \in \mathbb{Z}_{K_{n}}} \frac{T-\tau}{K_{n}} \log \left[1+\frac{\tau \delta \tilde{h}_{n, k}^{2} P_{n} K_{n}}{(T-\tau) \sigma}\right] .
\end{aligned}
$$

2) Uplink Information Transfer With 1-Bit CGR: For 1-bit CGR, we assume the AP allows only the SNs with $\tilde{b}_{n, k}=1$ to transmit in the uplink and excludes the rest of the SNs from transmitting. For $\beta_{n}$ number of transmitting SNs in the sector $\phi_{n}$, each SN transmits information in the uplink over a time duration of $(T-\tau) / \beta_{n}$ with the power of $\beta_{n} W_{n, k}\left(h_{n, k}, P_{n}\right) /(T-\tau)$. The uplink rate of $\mathbf{S N}_{n, k}$ is $\tilde{b}_{n, k} R_{n, k}\left(h_{n, k}, P_{n}, \beta_{n}\right)$, where $\tilde{b}_{n, k}$ indicates whether $\mathbf{S N}_{n, k}$ is allocated a sub-slot for the uplink transmission. From the AP's perspective, the achievable uplink sum rate for all SNs is given by

$$
R_{\text {sum }}\left(P_{n}, \beta_{n}\right)=(T-\tau) \sum_{n \in \mathbb{Z}_{N}} \log \left[1+\frac{\tau \delta \gamma^{2} P_{n} \beta_{n}}{(T-\tau) \sigma}\right] .
$$

\section{B. Optimal Power Allocation for Uplink Sum Rate Maximization}

Now we derive the optimal power allocation in the downlink to maximize the achievable sum rate of all SNs in the uplink subject to the AP's total transmit power constraint, i.e.,

$$
\begin{aligned}
\text { P5 : } & \max _{P_{n}} R_{\text {sum }} \\
\text { s.t. } & \sum_{n \in \mathbb{Z}_{N}} P_{n} \leq P_{\text {tot }} \\
& P_{n} \geq 0 .
\end{aligned}
$$

Since P5 is a concave optimization problem, there exists a unique optimal solution for P5. 
1) Optimal Power Allocation With Full CGR: For full CGR, the optimal power allocation $P_{n}^{*}\left(\tilde{h}_{n, k}\right)$ is a waterfilling solution similar to [2]. The closed-form solution can be obtained for $K_{n}=1$ and solved numerically for other cases.

2) Optimal Power Allocation With 1-Bit CGR: The optimal power allocation for uplink achievable sum rate maximization with 1-bit CGR is discussed as follows. If $\beta_{n}=0 \forall n$, we have $P_{n}^{*}=0 \forall n$. Otherwise, $P_{n}^{*}$ is given by the water filling solution, i.e.,

$$
P_{n}^{*}\left(\tilde{b}_{n, k} \gamma\right)=\left[\frac{T-\tau}{\mu}-\frac{\rho}{\gamma^{2} \beta_{n}}\right]^{+},
$$

where $\rho=\frac{(T-\tau) \sigma}{\tau \delta}$ and $\mu$ is the solution to $\sum_{n \in \mathbb{Z}_{N}}\left[\frac{T-\tau}{\mu}-\frac{\rho}{\gamma^{2} \beta_{n}}\right]^{+}=P_{t o t}$. The sector $\phi_{n}$ receives non-zero power from the AP if the sum of reported 1-bit CGR $\beta_{n}>\frac{\rho \mu}{(T-\tau) \gamma^{2}}$.

Since both $P_{n}^{*}\left(\tilde{h}_{n, k}\right)$ and $P_{n}^{*}\left(\tilde{b}_{n, k} \gamma\right)$ are non-decreasing with $\tilde{h}_{n, k}$ and $\tilde{b}_{n, k}$, respectively, in the Nash equilibrium, the reported channel gains for each SNs is $\tilde{h}_{n, k}^{*}=h_{\max }$ for full CGR and $\tilde{b}_{n, k}^{*}=1$ for 1-bit CGR, respectively, if no price function is used. Similar to the previous section, the false-reporting in the Nash equilibrium can greatly reduce the uplink sum rate compared with that of the truthful CGR. In the following two subsections, we design $C_{n, k}$ to ensure that the truthful reporting is the dominant strategy that maximizes the uplink utility of each SN.

\section{Uplink Price Function Design With Full CGR}

For full CGR, each $\mathrm{SN}_{n, k}$ decides on the optimal reported channel gain $\tilde{h}_{n, k}$ to maximize its own uplink rate under the optimal power allocation $P_{n}^{*}\left(\tilde{h}_{n, k}\right)$ and price function $C_{n, k}$, i.e.,

$$
\begin{aligned}
\text { P6 : } & \max _{\tilde{h}_{n, k}} U_{n, k}=R_{n, k}\left(h_{n, k}, P_{n}^{*}\left(\tilde{h}_{n, k}\right), K_{n}\right)-C_{n, k} \\
& \text { s.t. } 0 \leq \tilde{h}_{n, k} \leq h_{\max } .
\end{aligned}
$$

We adopt the VCG mechanism to design the price function $C_{n, k}$ for the uplink by replacing $W$ by $R$ in (14), where each $\mathrm{SN}_{n, k}$ pays the difference between the maximum achievable uplink sum rate of all other SNs in the network attributable to its presence. The optimal power allocation $P_{i}^{* *}$ and $P_{i}^{*}$ maximize the sum rate maximization without and with the participation of $\mathrm{SN}_{n, k}$, respectively. Under the VCG price, we can prove that truthful reporting of $\tilde{h}_{n, k}^{*}=h_{n, k}$ is the dominant strategy for each $\mathrm{SN}_{n, k}$ in P6. The proof is similar to Appendix A and is omitted here.

As a special case of $K_{i}=1$ for all $i \in \mathbb{Z}_{N}$, we denote $\mathbf{S N}_{i, j}$ as $\mathbf{S N}_{i}$ and $\tilde{h}_{i, j}$ as $\tilde{h}_{i}$. The VCG price becomes

$$
\begin{aligned}
C_{n}=(T-\tau)[ & \sum_{i \in \mathbb{Z}_{N}, i \neq n} \log \left(1+\frac{\tilde{h}_{i}^{2} P_{i}^{* *}}{\rho}\right) \\
& \left.-\sum_{i \in \mathbb{Z}_{N}, j \neq n} \log \left(1+\frac{\tilde{h}_{i}^{2} P_{i}^{*}\left(\tilde{h}_{n}\right)}{\rho}\right)\right],
\end{aligned}
$$

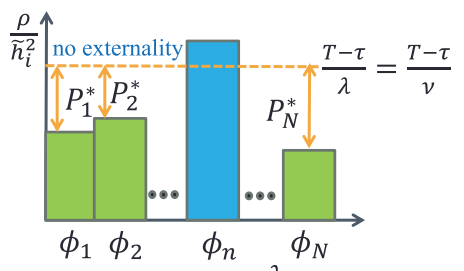

(a) $\tilde{h}_{n}^{2} \leq \frac{\rho \lambda}{T-\tau}$

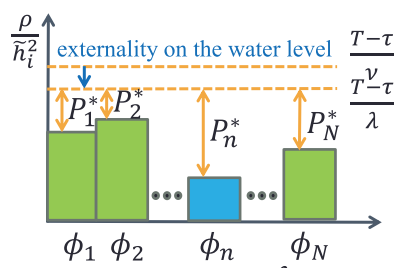

(b) $\tilde{h}_{n}^{2}>\frac{\rho \lambda}{T-\tau}$

Fig. 3. The externality of $\mathrm{SN}_{n}$ on the optimal power allocation in the system for $K_{n}=1$. (a) $\mathrm{SN}_{n}$ has no externality on other SNs if $\tilde{h}_{n}^{2} \leq \frac{\rho \lambda}{T-\tau}$; (b) $\mathrm{SN}_{n}$ has negative externality on other $\mathrm{SNs}$ if $\tilde{h}_{n}^{2}>\frac{\rho \lambda}{T-\tau}$.

where $P_{i}^{*}\left(\tilde{h}_{n}\right)$ and $P_{i}^{* *}$ are given by $P_{i}^{*}\left(\tilde{h}_{n}\right)=\left[\frac{T-\tau}{\lambda}-\frac{\rho}{h_{i}^{2}}\right]^{+}$ and $P_{i}^{* *}=\left[\frac{T-\tau}{\nu}-\frac{\rho}{h_{i}^{2}}\right]^{+}$. And the Lagrange multipliers $\lambda$ and $\nu$ are solved by $\sum_{n \in \mathbb{Z}_{N}}\left[\frac{T-\tau}{\lambda}-\frac{\rho}{h_{n}^{2}}\right]^{+}=P_{\text {tot }}$ and $\sum_{i \in \mathbb{Z}_{N}, i \neq n}\left[\frac{T-\tau}{\nu}-\frac{\rho}{\tilde{h}_{i}^{2}}\right]^{+}=P_{t o t}$, respectively.

Remark 6: Similar to (14), the VCG price in (33) is the externality that it imposes on other SNs, which can be reflected by the reduction of water level due to its participation. We illustrate this effect of the externality in Fig. 3. If $\tilde{h}_{n}^{2} \leq$ $\frac{\rho \lambda}{T-\tau}$ as shown in Fig. 3(a), we have $P_{n}^{*}=\left[\frac{T-\tau}{\lambda}-\frac{\rho}{h_{n}^{2}}\right]^{+}=0$. In this case, the water level remains the same without and with the participation of $\mathrm{SN}_{n}$, i.e., $\frac{T-\tau}{\nu}=\frac{T-\tau}{\lambda}$. In this case, $\mathrm{SN}_{n}$ pays zero price $C_{n}=0$ since it causes no externality on other SNs. If $\tilde{h}_{n}^{2}>\frac{\rho \lambda}{T-\tau}$ as shown in Fig. 3(b), $\mathrm{SN}_{n}$ receives non-zero power $P_{n}^{*}=\frac{T-\tau}{\lambda}-\frac{\rho}{h_{n}^{2}}>0$ and the water level reduces from $\frac{T-\tau}{\nu}$ to $\frac{T-\tau}{\lambda}$. The participation of $\mathrm{SN}_{n}$ reduces the received power (if non-zero) of all other SNs, i.e., $P_{i}^{*}\left(\tilde{h}_{n}\right) \leq P_{i}^{* *}$ for all $i \neq n$. In this case, $\mathrm{SN}_{n}$ needs to pay for the sum rate deduction of all other SNs in the system, which offsets its over-reporting incentive.

\section{Uplink Price Function Design With 1-Bit CGR}

For 1-bit CGR, each $\mathrm{SN}_{n, k}$ chooses the optimal reporting strategy $\tilde{b}_{n, k}$ to maximize its own utility $U_{n, k}$ under the efficient power allocation $P_{n}^{*}\left(\tilde{b}_{n, k} \gamma\right)$ and price function $C_{n, k}$, i.e.

$$
\begin{aligned}
\text { P7 : } & \max _{\tilde{b}_{n, k}} U_{n, k}=\tilde{b}_{n, k} R_{n, k}\left(h_{n, k}, P_{n}^{*}\left(\tilde{b}_{n, k} \gamma\right), \beta_{n}\right)-C_{n, k} \\
& \text { s.t. } \tilde{b}_{n, k} \in\{0,1\} .
\end{aligned}
$$

To design the price function for the 1-bit CGR, we also face the challenge that the information is asymmetric between the $\mathrm{AP}$ and the SNs, i.e., the SNs report only partial information of the channel gain to the AP. We will first prove that VCG mechanism fails to work, and then propose a threshold-based price function to ensure the truthfulness is the dominant strategy for each SN.

1) Failure of VCG: If we adopt VCG mechanism similar to the previous subsection, the price function of $\mathrm{SN}_{n, k}$ with 1-bit CGR equals to the total change of the sum of achievable rate 
of all other SNs in the system due to its participation, i.e.,

$$
\begin{aligned}
C_{n, k}= & \sum_{\substack{i \in \mathbb{Z}_{N} \\
i \neq n \\
i \neq n}} \sum_{j \in \mathbb{Z}_{K_{i}}} R_{i, j}\left(\tilde{b}_{i, j} \gamma, P_{i}^{* *}, \beta_{i}\right) \\
& +\sum_{\substack{j \in \mathbb{Z}_{K_{n}} \\
j \neq k}} R_{n, j}\left(\tilde{b}_{n, j} \gamma, P_{n}^{* *}, \hat{\beta}_{n}\right) \\
& -\sum_{\substack{i \in \mathbb{Z}_{N}, j \in \mathbb{Z}_{K_{i}} \\
(i, j) \neq(n, k)}} R_{i, j}\left(\tilde{b}_{i, j} \gamma, P_{i}^{*}\left(\tilde{b}_{n, k} \gamma\right), \beta_{i}\right) .
\end{aligned}
$$

Counterexample of VCG Mechanism: We now give an example to show that the VCG mechanism fails to guarantee the truthfulness of the 1-bit CGR for the uplink sum achievable rate maximization. Due to the participation of $\mathrm{SN}_{n, k}$, the sum of the achievable rate of other SNs may change because of two factors: (1) the change of power allocation across the sectors; (2) the change of time allocation within the sector $\phi_{n}$. We first ignore the effect of power allocation and focus on the effect of time allocation by considering $\beta_{n} \geq 2$ and $\beta_{i}=0 \forall i \neq n$. Since $\phi_{n}$ is the only sector with non-zero sum of reported CGR, the optimal power allocated to the sector $\phi_{n}$ without and with the participation of $\mathrm{SN}_{n, k}$ is the same, i.e., $P_{n}^{* *}=P_{n}^{*}=P_{\text {tot }}$. The VCG price in (36) becomes

$$
\begin{aligned}
C_{n, k}= & \sum_{j \in \mathbb{Z}_{K_{n}}, j \neq k} R_{n, j}\left(\tilde{b}_{n, j} \gamma, P_{t o t}, \hat{\beta}_{n}\right) \\
& -\sum_{j \in \mathbb{Z}_{K_{n}}, j \neq k} R_{n, j}\left(\tilde{b}_{n, j} \gamma, P_{t o t}, \beta_{n}\right) .
\end{aligned}
$$

We assume the true channel indicator of $\mathrm{SN}_{n, k}$ is $b_{n, k}=0$. By truthfully reporting with $\tilde{b}_{n, k}=b_{n, k}=0, \mathrm{SN}_{n, k}$ is not allowed to transmit in the uplink and its utility is $U_{n, k}=0$. By over-reporting with $\tilde{b}_{n, k}=1, \mathrm{SN}_{n, k}$ is allocated with a time fraction of $(T-\tau) / \beta_{n}$ to transmit in the uplink and we have $\beta_{n}=\hat{\beta}_{n}+1$. For other SNs in the sector $\phi_{n}$, their uplink transmission time reduces from $(T-\tau) / \hat{\beta}_{n}$ to $(T-\tau) / \beta_{n}$, which thus reduces their uplink rates. Using the VCG price in (37), the utility function of $\mathrm{SN}_{n, k}$ in (34) is rewritten as

$$
\begin{aligned}
U_{n, k}= & (T-\tau)\left[\frac{1}{\beta_{n}} \log \left(1+\frac{h_{n, k}^{2} P_{t o t} \beta_{n}}{\rho}\right)\right. \\
& \left.+\frac{\hat{\beta}_{n}}{\beta_{n}} \log \left(1+\frac{\gamma^{2} P_{t o t} \beta_{n}}{\rho}\right)-\log \left(1+\frac{\gamma^{2} P_{t o t} \hat{\beta}_{n}}{\rho}\right)\right] \\
> & \frac{T-\tau}{\beta_{n}}\left[\log \left(1+\frac{h_{n, k}^{2} P_{t o t} \beta_{n}}{\rho}\right)\right. \\
& \left.-\log \left(1+\frac{\gamma^{2} P_{t o t} \hat{\beta}_{n}}{\rho}\right)\right] .
\end{aligned}
$$

Based on $b_{n, k}=0$, we can only deduce that $h_{n, k}<\gamma$. However, if $h_{n, k}^{2} \beta_{n}>\gamma^{2} \hat{\beta}_{n}$, the right handside of (38) is greater than 0 and we have $U_{n, k}>0$. In this case, $\mathbf{S N}_{n, k}$ can still improve its own utility by over-reporting. From the above discussions, we see that VCG mechanism cannot always guarantee the truthfulness of the 1-bit CGR.
2) Threshold-Based Price Function: In this subsection, we design a threshold-based price function to guarantee the truthfulness of the 1-bit CGR from each SN.

Theorem 3: Under the optimal power allocation $P_{n}^{*}\left(\tilde{b}_{n, k} \gamma\right)$ for uplink sum rate maximization in (30), the threshold-based price function of

$$
C_{n, k}=\frac{\tilde{b}_{n, k}(T-\tau)}{\beta_{n}} \log \left(1+\frac{\tau \delta \gamma^{2} \beta_{n}}{(T-\tau) \sigma} P_{n}^{*}\left(\tilde{b}_{n, k} \gamma\right)\right),
$$

ensures that truthful-reporting of 1-bit channel gain (i.e., $\tilde{b}_{n, k}=b_{n, k}$ ) is the dominant strategy of each $\mathrm{SN}_{n, k}$ in P7.

Proof: If the true channel indicator of $\mathrm{SN}_{n, k}$ is $b_{n, k}=$ 0 , it implies $h_{n, k}<\gamma$. By truthfully reporting with $\tilde{b}_{n, k}=0$, we have $C_{n, k}=0$ and $U_{n, k}=0$. By overreporting with $\tilde{b}_{n, k}=1$, the utility of $\mathrm{SN}_{n, k}$ is $U_{n, k}=$ $\frac{T-\tau}{\beta_{n}} \log \left(1+\frac{\tau \delta h_{n, k}^{2} \beta_{n}}{(T-\tau) \sigma} P_{n}^{*}\right)-\frac{T-\tau}{\beta_{n}} \log \left(1+\frac{\tau \delta \gamma^{2} \beta_{n}}{(T-\tau) \sigma} P_{n}^{*}\right) \leq 0$ (the equality holds if $P_{n}^{*}=0$ ), which is no better than that of truthful reporting. If $b_{n, k}=1$, it implies that $h_{n, k} \geq \gamma$. By truthfully reporting with $\tilde{b}_{n, k}=1$, we have $U_{n, k}=$ $\frac{T-\tau}{\beta_{n}} \log \left(1+\frac{\tau \delta h_{n, k}^{2} \beta_{n}}{(T-\tau) \sigma} P_{n}^{*}\right)-\frac{T-\tau}{\beta_{n}} \log \left(1+\frac{\tau \delta \gamma^{2} \beta_{n}}{(T-\tau) \sigma} P_{n}^{*}\right) \geq 0$. By under-reporting with $\tilde{b}_{n, k}=0$, the utility of $\mathrm{SN}_{n, k}$ is $U_{n, k}=0$ which is also no better than that of truthful reporting. We thus prove that the price function in (39) ensures that truthful reporting is the dominant strategy for each SN.

Remark 7: From Theorem 3, we see that the threshold price that $\mathrm{SN}_{n, k}$ pays the amount of price that equals its own achievable rate in the uplink. Comparing the two thresholdbased price functions for the downlink in (23) and for the uplink in (39), we notice that there is no need to subtract the effect of other SNs in the uplink price since there is no free-riding effect. Different from the non-exclusive and nonrival energy transfer in the same energy beam, the information transmission is exclusive and rival, where each SN is allocated a dedicated time slot only if it reports $\tilde{b}_{n, k}=1$. Though $\mathrm{SN}_{n, k}$ with $\tilde{b}_{n, k}=0$ may also receive free energy from other co-sector SNs in the downlink, its utility is still zero due to the lack of transmission slot in the uplink.

\section{NumERicAl RESUlts}

In this section, we discuss the numerical results of the truthful mechanisms for the two CGR schemes. Without loss of generality, we consider a simple model with three SNs in two sectors, i.e., $\mathrm{SN}_{1,1}$ is in the sector $\phi_{1}, \mathrm{SN}_{2,1}$ and $\mathrm{SN}_{2,2}$ are in the sector $\phi_{2}$. We mainly discuss the price function design for $\mathrm{SN}_{2,2}$. We denote the parameters under the VCG mechanism and threshold-based mechanism by the superscripts of ${ }^{\mathrm{VCG}}$ and ${ }^{\mathrm{TH}}$. The uplink rate in this section is measured in nats/s/Hz.

In Fig. 4, we show that the VCG price in Proposition 1 guarantees the truthfulness of the full CGR from the SNs under the optimal power allocation for the downlink sum energy maximization. In Fig. 4(a) and Fig. 4(b), $\mathrm{SN}_{2,2}$ receives higher energy $W_{2,2}$ by reporting $\tilde{h}_{2,2} \geq 0.2$ than that of $\tilde{h}_{2,2}<0.02$. If there is no price, $\mathrm{SN}_{2,2}$ has the incentive to over-report its channel gain $\tilde{h}_{2,2}$ in order to increase its received energy. To offset the over-reporting incentive, the VCG mechanism 


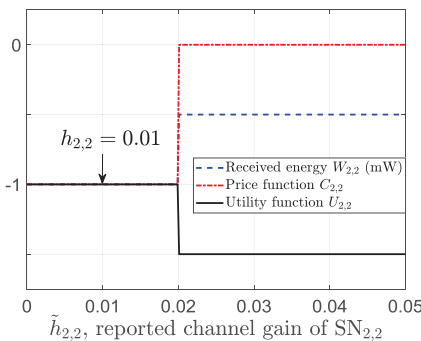

(a)

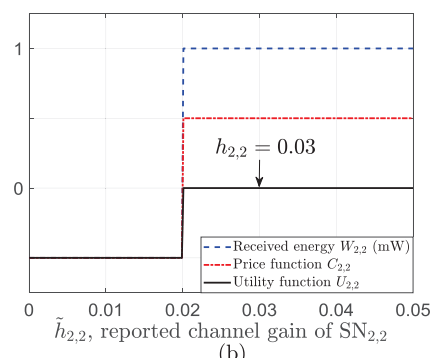

(b)
Fig. 4. The truthfulness of the VCG mechanism for the downlink sum energy maximization with full CGR $\left(h_{1,1}=0.04, h_{2,1}=0.02, P_{\text {tot }}=0.1 \mathrm{~W}\right.$, $\delta=1, \tau=1 \mathrm{~s}$ ). (a) The true valuatoin is $h_{2,2}=0.01$; (b) $h_{2,2}=0.03$.

\section{TABLE I}

COMPARISON BETWEen THE VCG AND THRESHOLD-BASED MECHANISMS FOR DOWNLINK SUM ENERGY MAXIMIZATION WITH 1-BIT CGR $\left(P_{\text {tot }}=0.1 \mathrm{~W}, \gamma=0.03, b_{1,1}=1\right.$, $\tilde{b}_{2,1}=1, h_{2,2}=0.02, b_{2,2}=0, \delta=1, \tau=1 \mathrm{~s}$.

\begin{tabular}{|c|c|c|}
\hline $\mathrm{SN}_{2,2}$ 's reported channel indicator & $\tilde{b}_{2,2}=0$ & $\tilde{b}_{2,2}=1$ \\
\hline$W_{2,2}($ received energy) & $1(\mathrm{~mW})$ & $2(\mathrm{~mW})$ \\
\hline$C_{2,2}^{\mathrm{VCG}}($ VCG price) & 0 & 0 \\
\hline$U_{2,2}^{\mathrm{VCG}}$ (utility with VCG price) & 1 & 2 \\
\hline$C_{2,2}^{\mathrm{TH}}$ (threshold-based price) & 0 & 1.5 \\
\hline$U_{2,2}^{\mathrm{TH}}$ (utility with threshold-based price) & 1 & 0.5 \\
\hline
\end{tabular}

requires $\mathrm{SN}_{2,2}$ to pay the loss of the sum received energy of $\mathrm{SN}_{1,1}$ and $\mathrm{SN}_{2,1}$ due to its participation. In both figures, $\mathrm{SN}_{2,2}$ cannot get better off by either over-reporting or underreporting. Therefore, it will truthfully report under the VCG price.

In Table 1, we give an example to show that the thresholdbased price in Theorem 2 ensures the truthfulness of the 1-bit CGR while the VCG price fails under the optimal power allocation that maximizes the achievable sum energy. We assume the truthful channel indicator for $\mathrm{SN}_{2,2}$ is $b_{2,2}=0$. By over-reporting with $\tilde{b}_{n, k}=1, \mathrm{SN}_{2,2}$ receives more energy of $W_{2,2}$. We need to see whether the two price functions can successfully offset this over-reporting incentive. Using the VCG price, $\mathrm{SN}_{2,2}$ pays zero price regardless of its reported channel gains so that it gains more utility by over-reporting than by truthfully reporting. Using the threshold-based price, $\mathrm{SN}_{2,2}$ pays a positive price of 1.5 if it over-reports with $\tilde{b}_{2,2}=1$ and zero price if it truthfully reports with $\tilde{b}_{2,2}=0$. As a result, the sensor gains higher utility by truthfully reporting under the threshold-based price.

Fig. 5 illustrates that the VCG price guarantees the truthfulness of the full CGR from the SNs under the optimal power allocation for the uplink sum rate maximization. In Fig. 5(a), the uplink rate $R_{2,2}$ increases as the increase of the reported channel gain $\tilde{h}_{2,2}$, which motivates $\mathrm{SN}_{2,2}$ to over-report. To compensate the over-reporting incentive, the VCG price $C_{2,2}$ also increases with $\tilde{h}_{2,2}$, where the increasing slope of $C_{2,2}$ is sharper than that of $R_{2,2}$ for $\tilde{h}_{2,2}>h_{2,2}$. As a result, the maximum value of $U_{2,2}$ is achieved at the point where $\mathrm{SN}_{2,2}$ truthfully reports its channel gain, i.e., $\tilde{h}_{2,2}=h_{2,2}$. In Fig. 5(b), we further show that truthful reporting is the
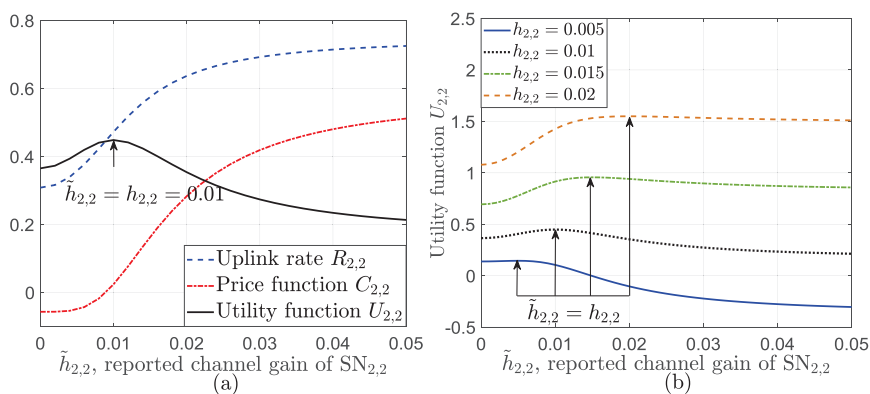

Fig. 5. Truthfulness of the VCG mechanism for the uplink sum rate maximization with full CGR. Consider $\mathrm{SN}_{1,1}$ is in the sector $\phi_{1}, \mathrm{SN}_{2,1}$ and $\mathrm{SN}_{2,2}$ are in the sector $\phi_{2}\left(\tilde{h}_{1,1}=0.02, \tilde{h}_{2,1}=0.02, P_{\text {tot }}=0.1 \mathrm{~W}\right.$, $\delta=1, \sigma=10^{-5} \mathrm{~W}, \tau=1 \mathrm{~s}, T=5 \mathrm{~s}$ ). (a) With full CGR, $\mathrm{SN}_{2,2}$ 's uplink rate $R_{2,2}$, price function $C_{2,2}$, and utility function $U_{2,2}$ versus the $\mathrm{SN}_{2,2}$ 's reported channel gain $\tilde{h}_{2,2}$; (b) $\mathrm{SN}_{2,2}$ 's utility function versus the $\mathrm{SN}_{2,2}$ 's reported channel gain $\tilde{h}_{2,2}$ under different true channel gain $h_{2,2}$.

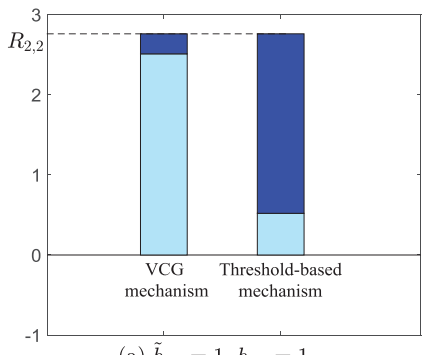

(a) $\tilde{b}_{2,2}=1, b_{2,2}=1$

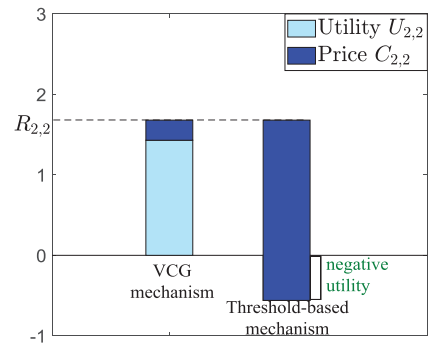

(b) $\tilde{b}_{2,2}=1, b_{2,2}=0$
Fig. 6. Comparison between the VCG and threshold-based mechanisms for uplink sum rate maximization with 1-bit CGR. Consider $\mathrm{SN}_{1,1}$ is in the sector $\phi_{1}, \mathrm{SN}_{2,1}$ and $\mathrm{SN}_{2,2}$ are in the sector $\phi_{2}\left(\tilde{h}_{1,1}=0.04, \tilde{h}_{2,1}=0.04\right.$ $\left.P_{\text {tot }}=0.1 \mathrm{~W}, \gamma=0.025, \delta=1, \sigma=10^{-5} \mathrm{~W}, \tau=1 \mathrm{~s}, T=5 \mathrm{~s}\right)$. (a) $h_{2,2}=0.03$; (b) $h_{2,2}=0.02$.

dominant strategy of $\mathrm{SN}_{2,2}$ that maximizes its uplink utility for various values of $h_{2,2}$.

In Fig. 6, we show that the threshold-based price function in Theorem 3 guarantees the truthfulness of the 1-bit CGR but the VCG mechanism in (37) is not always truthful under the optimal power allocation that maximizes the achievable sum rate. Firstly, we can easily deduce that $\mathrm{SN}_{2,2}$ has zero utility if it reports $\tilde{b}_{2,2}=0$ under either price function, regardless of its truthful channel valuation. By reporting $\tilde{b}_{2,2}=1, \mathbf{S N}_{2,2}$ obtains a positive uplink rate and also pays a positive price to the AP, where the corresponding VCG price is $C_{2,2}^{\mathrm{VCG}}\left(\tilde{b}_{2,2}=1\right)=0.25$ and threshold-based price is $C_{2,2}^{\mathrm{TH}}\left(\tilde{b}_{2,2}=1\right)=2.24$. Next, we discuss whether the two price functions ensure the truthfulness of the 1-bit CGR. When the truthful channel indicator of $\mathrm{SN}_{2,2}$ is $b_{2,2}=1$ as shown in Fig. 6(a), the utility functions are $U_{2,2}^{\mathrm{VCG}}\left(\tilde{b}_{2,2}=1\right)=2.51$ and $U_{2,2}^{\mathrm{TH}}\left(\tilde{b}_{2,2}=1\right)=0.52$, which are both higher than that of under-reporting with $\tilde{b}_{2,2}=0$. In this case, $\mathrm{SN}_{2,2}$ has no incentive to cheat under either mechanisms. When the truthful channel indicator is $b_{2,2}=0$ as shown in Fig. 6(b), the utility functions of $\mathrm{SN}_{2,2}$ under the VCG price and threshold-based price are $U_{2,2}^{\mathrm{VCG}}\left(\tilde{b}_{2,2}=1\right)=1.43$ and $U_{2,2}^{\mathrm{TH}}\left(\tilde{b}_{2,2}=1\right)=-0.56$, respectively, which is higher than and smaller than that of the truthful reporting, respectively. In this case, $\mathrm{SN}_{2,2}$ will 
cheat under the VCG price while reporting honestly under the threshold-based price.

\section{CONCLUSION}

In this paper, we considered a harvest-then-transmit directional WPT system, where the AP allocates the network resources based on the SNs' full or binary reported channel gains. We first derived the AP's optimal power allocation that maximizes the social welfare, i.e., downlink sum energy and the uplink sum rate of all SNs, respectively. Under such power allocation, we proved that the self-interested SNs have the incentives to exaggerate their channel gains to increase their own utilities, which reduces the social welfare compared with that of the truthful reporting. To align the locally optimal strategies of the SNs with the maximum social welfare, we designed the price functions to offset the over-reporting incentives so that truthful reporting is the dominant strategy for each SN. Under the full CGR scheme, we derived the price functions based on the VCG mechanism for the downlink and uplink, respectively. Under the 1-bit CGR scheme, we first proved that the VCG mechanism is not truthful and then proposed the threshold-based price functions for the downlink and uplink, respectively. Finally, we use numerical results to further validate the truthfulness of the our mechanism designs. In the future work, we are working towards generalizing the mechanism design into the multi-bit channel feedback.

\section{APPENDIX A}

As discussed, the optimal power allocated to the sector $\phi_{i}$ with and without the participation of $\mathrm{SN}_{n, k}$ are given by $P_{i}^{*}$ and $P_{i}^{* *}$, which are the solutions to $\mathrm{P} 1$ and $\mathrm{P} 4$, respectively. Under the VCG price and efficient power allocation, each selfinterested $\mathbf{S N}_{n, k}$ chooses the optimal reported channel gain $h_{n, k}$ to maximize its utility function $U_{n, k}$ in (11), i.e.,

$$
\begin{aligned}
\max _{\tilde{h}_{n, k}} U_{n, k}= & \max _{\tilde{h}_{n, k}}\left\{W_{n, k}\left(h_{n, k}, P_{n}^{*}\left(\tilde{h}_{n, k}\right)\right)-C_{n, k}\right\} \\
= & \max _{\tilde{h}_{n, k}}\left\{W_{n, k}\left(h_{n, k}, P_{n}^{*}\left(\tilde{h}_{n, k}\right)\right)\right. \\
& -\left\{\sum_{\substack{i \in \mathbb{Z}_{N}, j \in \mathbb{Z}_{K_{n}} \\
(i, j) \neq(n, k)}} W_{i, j}\left(\tilde{h}_{i, j}, P_{i}^{* *}\right)\right. \\
& \left.\left.-\sum_{\substack{i \in \mathbb{Z}_{N}, j \in \mathbb{Z}_{K_{n}} \\
(i, j) \neq(n, k)}} W_{i, j}\left(\tilde{h}_{i, j}, P_{i}^{*}\left(\tilde{h}_{n, k}\right)\right)\right\}\right\} \\
= & \max _{\tilde{h}_{n, k}}\left\{W_{n, k}\left(h_{n, k}, P_{n}^{*}\left(\tilde{h}_{n, k}\right)\right)\right. \\
& \left.+\sum_{\substack{i \in \mathbb{Z}_{N}, j \in \mathbb{Z}_{K_{n}} \\
(i, j) \neq(n, k)}} W_{i, j}\left(\tilde{h}_{i, j}, P_{i}^{*}\left(\tilde{h}_{n, k}\right)\right)\right\} \\
& -\sum_{\substack{n \in \mathbb{Z}_{N}, k \in \mathbb{Z}_{K_{n}} \\
(i, j) \neq(n, k)}} W_{i, j}\left(\tilde{h}_{i, j}, P_{i}^{* *}\right) .
\end{aligned}
$$

The last equality holds since $\sum \sum_{n \in \mathbb{Z}_{N}, k \in \mathbb{Z}_{K_{n}}} W_{i, j}$ $\left(\tilde{h}_{i, j}, P_{i}^{* *}\right)$ does not depend on $\tilde{h}_{n, k} \stackrel{(i, j) \neq(n, k)}{\text { Recall }}$ that $P_{n}^{*}=\arg \max _{P_{n}} W_{\text {sum }}\left(P_{n}\right)=\arg \max _{P_{n}}\left[W_{n, k}\left(\tilde{h}_{n, k}, P_{n}\left(\tilde{h}_{n, k}\right)\right)+\right.$ $\left.\sum \sum \sum_{i \in \mathbb{Z}_{N}, j \in \mathbb{Z}_{K_{n}}} W_{i, j}\left(\tilde{h}_{i, j}, P_{i}\left(\tilde{h}_{n, k}\right)\right)\right]$ which depends on the $(i, j) \neq(n, k)$

reported channel gain $\tilde{h}_{n, k}$. If possible, $\mathbf{S N}_{n, k}$ would pick a reported channel gain $\tilde{h}_{n, k}$ that leads the mechanism to pick a $P_{n}$ which solves

$$
\begin{aligned}
\max _{P_{n}}\left\{W _ { n , k } \left(h_{n, k},\right.\right. & \left.P_{n}\left(\tilde{h}_{n, k}\right)\right) \\
& \left.+\sum_{\substack{i \in \mathbb{Z}_{N}, j \in \mathbb{Z}_{K_{n}} \\
(i, j) \neq(n, k)}} W_{i, j}\left(\tilde{h}_{i, j}, P_{i}\left(\tilde{h}_{n, k}\right)\right)\right\} .
\end{aligned}
$$

The VCG mechanism will choose $P_{n}$ in a way that solves the maximization problem in (41) when $\mathrm{SN}_{n, k}$ reports $\tilde{h}_{n, k}=h_{n, k}$. Since this argument does not depend on other SNs' strategies, truthful reporting is the dominant strategy for each $\mathrm{SN}_{n, k}$.

\section{APPENDIX B}

By substituting the optimal power allocation in (7) and the price function in (21) into the utility function in (11), we have

$U_{n, k}=\left\{\begin{array}{c}\tau \delta P_{t o t} h_{n, k} / M, \quad \text { if } \alpha_{n}=\alpha_{(N)} \text { and } \hat{\alpha}_{n}=\hat{\alpha}_{(N)} \\ \tau \delta P_{t o t}\left[\left(h_{n, k}-\tilde{h}_{n, k}\right) / M-\hat{\alpha}_{(N)}+\alpha_{(N)}\right] \\ \text { if } \alpha_{n}=\alpha_{(N)} \text { and } \hat{\alpha}_{n}<\hat{\alpha}_{(N)} \\ 0, \text { if } \alpha_{n}<\alpha_{(N)} .\end{array}\right.$

Case 1: $h_{n, k}+\hat{\alpha}_{n}<\hat{\alpha}_{(N)}$. 1) Consider $\mathrm{SN}_{2}$ truthfully reports with $\tilde{h}_{n, k}=h_{n, k}$. In this case, we have $\alpha_{n}=$ $\tilde{h}_{n, k}+\hat{\alpha}_{n}=h_{n, k}+\hat{\alpha}_{n}<\alpha_{(N)}$. According to (42c), we have $\left.U_{n, k}=0.2\right)$ Consider $\mathrm{SN}_{2}$ over-reports with $\tilde{h}_{n, k}>h_{n, k}$. If $\alpha_{n}=\alpha_{(N)}$ (with $M=1$ ), we have $\hat{\alpha}_{n}=\alpha_{n}-$ $\tilde{h}_{n, k}=\alpha_{(N)}-\tilde{h}_{n, k}$. According to (42b), we have $U_{n, k}=$ $\tau \delta P_{t o t}\left[h_{n, k}-\hat{\alpha}_{(N)}+\alpha_{(N)}-\tilde{h}_{n, k}\right]=\tau \delta P_{t o t}\left[h_{n, k}-\hat{\alpha}_{(N)}+\right.$ $\left.\hat{\alpha}_{n}\right]<0$, which is worse than that of truthful reporting. If $\alpha_{n}=\alpha_{(N)}$ (with $M \geq 2$ ), we can easily deduce that $\alpha_{(N)}=\hat{\alpha}_{(N)}$. According to (42b), we have $U_{n, k}=$ $\tau \delta P_{t o t}\left[\left(h_{n, k}-\tilde{h}_{n, k}\right) / M-\hat{\alpha}_{(N)}+\alpha_{(N)}\right]=\tau \delta P_{t o t}\left(h_{n, k}-\right.$ $\left.\tilde{h}_{n, k}\right) / M<0$, which is worse than that of truthful reporting. If $\alpha_{n}<\alpha_{(N)}$, we have $U_{n, k}=0$ according to (42c). 3) Consider $\mathrm{SN}_{3}$ under-reports with $\tilde{h}_{n, k}<h_{n, k}$. In this case, we have $\alpha_{n}=\tilde{h}_{n, k}+\hat{\alpha}_{n}<h_{n, k}+\hat{\alpha}_{n}<\hat{\alpha}_{(N)}$ and thus have $\alpha_{n}<\alpha_{(N)}$. According to (42c), we have $U_{n, k}=0$, which is the same as truthful reporting.

Case 2: $h_{n, k}+\hat{\alpha}_{n} \geq \hat{\alpha}_{(N)}$ and $\hat{\alpha}_{n}<\hat{\alpha}_{(N)}$. 1) Consider $\mathrm{SN}_{n, k}$ truthfully reports with $\tilde{h}_{n, k}=h_{n, k}$. In this case, we have $\alpha_{n}=\tilde{h}_{n, k}+\hat{\alpha}_{n}=h_{n, k}+\hat{\alpha}_{n} \geq \hat{\alpha}_{(N)}$ and thus can deduce that $\alpha_{n}=\alpha_{(N)}=\tilde{h}_{n, k}+\hat{\alpha}_{n}=h_{n, k}+\hat{\alpha}_{n}$. According to (42b), we have $U_{n, k}=\tau \delta P_{\text {tot }}\left[\left(h_{n, k}-\tilde{h}_{n, k}\right) / M+\alpha_{(N)}-\right.$ $\left.\hat{\alpha}_{(N)}\right]=\tau \delta P_{t o t}\left[\alpha_{(N)}-\hat{\alpha}_{(N)}\right]=\tau \delta P_{t o t}\left[h_{n, k}+\hat{\alpha}_{n}-\hat{\alpha}_{(N)}\right] \geq 0$. 2) Consider $\mathrm{SN}_{2}$ over-reports with $\tilde{h}_{n, k}>h_{n, k}$. In this case, we have $\alpha_{n}=\tilde{h}_{n, k}+\hat{\alpha}_{n}>h_{n, k}+\hat{\alpha}_{n} \geq \hat{\alpha}_{(N)}$ and thus have $\alpha_{n}=\alpha_{(N)}$ (with $M=1$ ) and $\tilde{h}_{n, k}=\alpha_{(N)}-\hat{\alpha}_{n}$. According to (42b), we have $U_{n, k}=\tau \delta P_{t o t}\left[h_{n, k}+\alpha_{(N)}-\right.$ $\left.\tilde{h}_{n, k}-\hat{\alpha}_{(N)}\right]=\tau \delta P_{t o t}\left[h_{n, k}+\hat{\alpha}_{n}-\hat{\alpha}_{(N)}\right] \geq 0$, which is the same as that of truthful reporting. 3) Consider $\mathrm{SN}_{3}$ underreports with $\tilde{h}_{n, k}<h_{n, k}$. If $h_{n, k}+\hat{\alpha}_{n}>\tilde{h}_{n, k}+\hat{\alpha}_{n} \geq \hat{\alpha}_{(N)}$, 
we have $\alpha_{n}=\alpha_{(N)}$ and thus have $\tilde{h}_{n, k}=\alpha_{(N)}-\hat{\alpha}_{n}$. Since $M \geq 1$ and $h_{n, k}-\tilde{h}_{n, k}>0$, we have $U_{n, k}=$ $\tau \delta P_{t o t}\left[\left(h_{n, k}-\tilde{h}_{n, k}\right) / M-\hat{\alpha}_{(N)}+\alpha_{(N)}\right] \leq \tau \delta P_{t o t}\left[h_{n, k}-\right.$ $\left.\tilde{h}_{n, k}-\hat{\alpha}_{(N)}+\alpha_{(N)}\right]=\tau \delta P_{t o t}\left[h_{n, k}+\hat{\alpha}_{n}-\hat{\alpha}_{(N)}\right]$. In this case, under-reporting is worse than or at most equivalent to truthful reporting. If $h_{n, k}+\hat{\alpha}_{n} \geq \hat{\alpha}_{(N)}>\tilde{h}_{n, k}+\hat{\alpha}_{n}$, we have $\alpha_{n}=\tilde{h}_{n, k}+\hat{\alpha}_{n}<\hat{\alpha}_{(N)} \leq \alpha_{(N)}$. According to (42c), we have $U_{n, k}=0$ which is worse than that of truthful reporting.

Case 3: $h_{n, k}+\hat{\alpha}_{n} \geq \hat{\alpha}_{(N)}$ and $\hat{\alpha}_{n}=\hat{\alpha}_{(N)}$. In this case, we can deduce that $\alpha_{n}=\alpha_{(N)}$. According to (42a), we have $U_{n, k}=\tau \delta P_{t o t} h_{n, k} / M$ for any reported channel gain of $\tilde{h}_{n, k}$.

From the above discussions, we see that $\mathrm{SN}_{n, k}$ cannot get better off by either over-reporting or under-reporting, regardless of other SNs' reporting strategies. Therefore, truthful reporting is the weakly dominant strategy for each SN. Furthermore, the individual rational holds as the utility of a truthful SN is always non-negative under its dominant strategy. If an $\mathrm{SN}$ does not participate in the game, it is equivalent to under-reporting $\tilde{h}_{n, k}=0$, where the $\mathrm{SN}$ will have less utility than truthful reporting.

\section{APPENDIX C}

Substituting (23) into (11), the utility function of $\mathrm{SN}_{n, k}$ is

$$
U_{n, k}=\tau \delta\left[h_{n, k} P_{n}^{*}-\tilde{b}_{n, k} \gamma\left(P_{n}^{*}-P_{n}^{* *}\right)\right] .
$$

Denote $\Delta=P_{n}^{*}-P_{n}^{* *}$, it can be easily proved that $\Delta \geq 0$. Substituting $\Delta$ into (43), we have

$$
\begin{aligned}
U_{n, k} & =\tau \delta\left[h_{n, k}\left(P_{n}^{* *}+\Delta\right)-\tilde{b}_{n, k} \gamma \Delta\right] \\
& =\tau \delta\left[h_{n, k} P_{n}^{* *}+\left(h_{n, k}-\tilde{b}_{n, k} \gamma\right) \Delta\right] .
\end{aligned}
$$

If $b_{n, k}=0$, we have $h_{n, k}<\gamma$. By truthfully reporting with $\tilde{b}_{n, k}=0$, we have $P_{n}^{*}=P_{n}^{* *}$ and its utility in (43) is $U_{n, k}=\tau \delta h_{n, k} P_{n}^{*}=\tau \delta h_{n, k} P_{n}^{* *}$. By over-reporting with $\tilde{b}_{n, k}=1$, we have

$$
U_{n, k}=\tau \delta\left[h_{n, k} P_{n}^{* *}+\left(h_{n, k}-\tilde{b}_{n, k} \gamma\right) \Delta\right]<\tau \delta h_{n, k} P_{n}^{* *}
$$

which is worse than that of truthful reporting.

If $b_{n, k}=1$, we have $h_{n, k} \geq \gamma$. By truthfully reporting with $\tilde{b}_{n, k}=1$, the utility of $\mathrm{SN}_{n, k}$ is

$$
U_{n, k}=\tau \delta\left[h_{n, k} P_{n}^{* *}+\left(h_{n, k}-\tilde{b}_{n, k} \gamma\right) \Delta\right] \geq \tau \delta h_{n, k} P_{n}^{* *}
$$

By under-reporting with $\tilde{b}_{n, k}=0$, we have $P_{n}^{*}=P_{n}^{* *}$ and its utility in (43) is $U_{n, k}=\tau \delta h_{n, k} P_{n}^{*}=\tau \delta h_{n, k} P_{n}^{* *}$ which is no better than of truthful reporting.

To sum up, adopting the price function in (23), $\mathrm{SN}_{n, k}$ cannot improve its utility by deviating from the truthful reporting, regardless of other SNs' reporting strategies. We thus proved that the truthful reporting is the dominant strategy for each SN. Each SN is individual rational as it always has non-negative utility under its dominant strategy. If an SN does not participate in the game, it is equivalent to under-reporting $\tilde{b}_{n, k}=0$, where the SN will have less utility than truthful reporting.

\section{REFERENCES}

[1] P. Kamalinejad, C. Mahapatra, Z. Sheng, S. Mirabbasi, V. C. M. Leung, and Y. L. Guan, "Wireless energy harvesting for the Internet of Things," IEEE Commun. Mag., vol. 53, no. 6, pp. 102108, Jun. 2015.

[2] R. Zhang and C. K. Ho, "MIMO broadcasting for simultaneous wireless information and power transfer," IEEE Trans. Wireless Commun., vol. 12, no. 5, pp. 1989-2001, May 2013.

[3] K. Huang and V. K. N. Lau, "Enabling wireless power transfer in cellular networks: Architecture, modeling and deployment," IEEE Trans. Wireless Commun., vol. 13, no. 2, pp. 902-912, Feb. 2014.

[4] Z. Wang, L. Duan, and R. Zhang, "Adaptively directional wireless power transfer for large-scale sensor networks," IEEE J. Sel. Areas Commun., vol. 34, no. 5, pp. 1785-1800, May 2016.

[5] J. Guo, X. Zhou, and S. Durrani, "Wireless power transfer via mmWave power beacons with directional beamforming," IEEE Commun. Lett., vol. 8, no. 1, pp. 17-20, Feb. 2019.

[6] G. Yang, C. K. Ho, and Y. L. Guan, "Dynamic resource allocation for multiple-antenna wireless power transfer," IEEE Trans. Signal Process., vol. 62, no. 14, pp. 3563-3577, Jul. 2014.

[7] X. Chen, C. Yuen, and Z. Zhang, "Wireless energy and information transfer tradeoff for limited-feedback multiantenna systems with energy beamforming," IEEE Trans. Veh. Technol., vol. 63, no. 1, pp. 407-412, Jan. 2014

[8] X. Zhou, "Training-based SWIPT: Optimal power splitting at the receiver," IEEE Trans. Veh. Technol., vol. 64, no. 9, pp. 4377-4382, Sep. 2015.

[9] Y. Zeng and R. Zhang, "Optimized training design for wireless energy transfer," IEEE Trans. Commun., vol. 63, no. 2, pp. 536-550, Feb. 2015.

[10] S. Borade and L. Zheng, "Wideband fading channels with feedback," IEEE Trans. Inf. Theory, vol. 56, no. 12, pp. 6058-6065, Dec. 2010.

[11] J. Xu and R. Zhang, "Energy beamforming with one-bit feedback," IEEE Trans. Signal Process., vol. 62, no. 20, pp. 5370-5381, Oct. 2016.

[12] I. Krikidis, "Opportunistic beamforming with wireless powered 1-bit feedback through rectenna array," IEEE Signal Process. Lett., vol. 22, no. 11, pp. 2054-2058, Nov. 2015.

[13] A. Mas-Colell, M. D. Whinston, and J. R. Green, Microeconomic Theory. London, U.K.: Oxford Univ. Press, 1995.

[14] B. Wang, Y. Wu, Z. Ji, K. J. R. Liu, and T. C. Clancy, "Game theoretical mechanism design methods," IEEE Signal Process. Mag., vol. 25, no. 6, pp. 74-84, Nov. 2008.

[15] W. Vickrey, "Counterspeculation, auctions, and competitive sealed tenders," J. Finance, vol. 16, no. 1, pp. 8-37, Mar. 1961.

[16] E. H. Clarke, "Multipart pricing of public goods," Public Choice, vol. 11, no. 1, pp. 17-33, Sep. 1971.

[17] T. Groves, "Incentives in teams," Econometrica, vol. 41, no. 4, pp. 617-631, Jul. 1973.

[18] P. Milgrom, Putting Auction Theory to Work. Cambridge, U.K.: Cambridge Univ. Press, 2004.

[19] L. Anderegg and S. Eidenbenz, "Ad hoc-VCG: A truthful and cost-efficient routing protocol for mobile ad hoc networks with selfish agents," in Proc. 9th Аnnu. Int. Conf. Mobile Comput. Netw. (MobiCom), San Diego, CA, USA, Sep. 2003, pp. 245-259.

[20] J. Dai, F. Liu, B. Li, B. Li, and J. Liu, "Collaborative caching in wireless video streaming through resource auctions," IEEE J. Sel. Areas Commun., vol. 30, no. 2, pp. 458-466, Feb. 2012.

[21] C. Li, Y. Fu, Z. Liu, X.-Y. Liu, W. Wu, and L. Xiong, "Spectrum trading for energy-harvesting-enabled Internet of Things in harsh environments," IEEE Access, vol. 6, pp. 16712-16726, 2018.

[22] D. Niyato and P. Wang, "Competitive wireless energy transfer bidding: A game theoretic approach," in Proc. IEEE Int. Conf. Commun. (ICC), Sydney, NSW, Australia, May 2014, pp. 1-6.

[23] H. Chen, Y. Li, Z. Han, and B. Vucetic, "A Stackelberg gamebased energy trading scheme for power beacon-assisted wirelesspowered communication," in Proc. IEEE Int. Conf. Acoust., Speech Signal Process. (ICASSP), Brisbane, QLD, Australia, Apr. 2015, pp. 3177-3181.

[24] Y. Ma, H. Chen, Z. Lin, Y. Li, and B. Vucetic, "Distributed and optimal resource allocation for power beacon-assisted wireless-powered communications," IEEE Trans. Commun., vol. 63, no. 10, pp. 3569-3583, Oct. 2015

[25] S. Sarma, K. Kandhway, and J. Kuri, "Robust energy harvesting based on a Stackelberg game," IEEE Commun. Lett., vol. 5, no. 3, pp. 336-339, Jun. 2016. 
[26] M. Zhang, J. Huang, and R. Zhang, "Wireless power provision as a public good," in Proc. 16th Int. Symp. Modeling Optim. Mobile, Ad Hoc, Wireless Netw. (WiOpt), Shanghai, China, May 2018, pp. 1-8.

[27] Z. Hou, H. Chen, Y. Li, and B. Vucetic, "Incentive mechanism design for wireless energy harvesting-based Internet of Things," IEEE Internet Things J., vol. 5, no. 4, pp. 2620-2632, Aug. 2018.

[28] D. J. Love, R. W. Heath, Jr., V. K. Lau, D. Gesbert, B. D. Rao, and M. Andrews, "An overview of limited feedback in wireless communication systems," IEEE J. Sel. Areas Commun., vol. 26, no. 8, pp. 1341-1365, Oct. 2008.

[29] P. Xia and G. B. Giannakis, "Design and analysis of transmitbeamforming based on limited-rate feedback," IEEE Trans. Signal Process., vol. 54, no. 5, pp. 1853-1863, May 2006.

[30] H. Ju and R. Zhang, "Throughput maximization in wireless powered communication networks," IEEE Trans. Wireless Commun., vol. 13, no. 1, pp. 418-428, Jan. 2014.

[31] I. Krikidis, "Average age of information in wireless powered sensor networks," IEEE Wireless Commun. Lett., vol. 8, no. 2, pp. 628-631, Apr. 2019.

[32] E. Boshkovska, D. W. K. Ng, N. Zlatanov, and R. Schober, "Practical non-linear energy harvesting model and resource allocation for SWIPT systems," IEEE Commun. Lett., vol. 19, no. 12, pp. 2082-2085, Dec. 2015.

[33] R.-R. Chen, R. Koetter, U. Madhow, and D. Agrawal, "Joint noncoherent demodulation and decoding for the block fading channel: A practical framework for approaching Shannon capacity," IEEE Trans. Commun., vol. 51, no. 10, pp. 1676-1689, Oct. 2003.

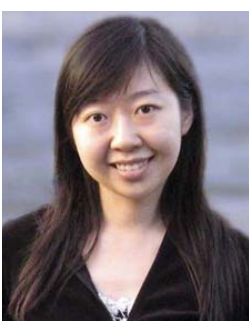

Zhe Wang (S'10-M'14) received the Ph.D. degree in electrical engineering from the University of New South Wales, Sydney, Australia, in 2014.

From 2015 to 2017, she was a Research Fellow with the Department of Electrical and Electronic Engineering, The University of Melbourne, Australia. She is currently a Research Fellow with the Engineering Systems and Design Pillar, Singapore University of Technology and Design, Singapore. Her research interests include resource allocation and optimization for wireless communications and networking.

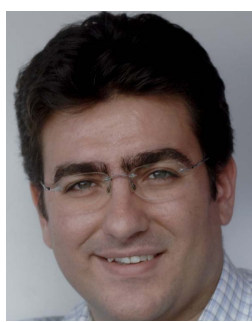

Tansu Alpcan (SM'13) received the Ph.D. degree in electrical and computer engineering from the University of Illinois at Urbana-Champaign (UIUC), in 2006.

$\mathrm{He}$ has worked as a Senior Research Scientist with the Deutsche Telekom Laboratories, Berlin, Germany, from 2006 to 2009, and as an Assistant Professor (Juniorprofessur) with Technical University, Berlin, from 2009 to 2011. He is currently with the Department of Electrical and Electronic Engineering, The University of Melbourne, as an Associate Professor and a Reader. He is the (co-)author of more than 150 journal and conference articles and the book Network Security: A Decision and Game Theoretic Approach [Cambridge University Press (CUP), 2011]. His research interests include applications of control, optimization, game theories, machine learning to security and resource allocation problems in communications, smart grid, and the Internet of Things.

Dr. Alpcan chaired or was an Associate Editor, the TPC chair, or a TPC member of several prestigious IEEE workshops, conferences, and journals. He co-edited the book Mechanisms and Games for Dynamic Spectrum Allocation (CUP, 2014)

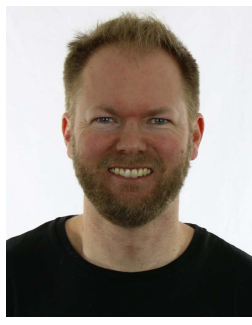

Jamie S. Evans (S'93-M'98-SM'17) was born in Newcastle, Australia, in 1970. He received the B.S. degree in physics and the B.E. degree in computer engineering from The University of Newcastle, in 1992 and 1993, respectively, where he received the University Medal upon graduation, and the M.S. and $\mathrm{Ph} . \mathrm{D}$. degrees in electrical engineering from The University of Melbourne, Australia, in 1996 and 1998, respectively, and was awarded the Chancellor's Prize for excellence for his Ph.D. dissertation.

From March 1998 to June 1999, he was a Visiting Researcher with the Department of Electrical Engineering and Computer Science, University of California at Berkeley. Since returning to Australia in July 1999, he has held an Academic positions at the University of Sydney, The University of Melbourne, and Monash University. He is currently a Professor and the Deputy Dean of the Melbourne School of Engineering, The University of Melbourne. His research interests are in communications theory, information theory, and statistical signal processing with a focus on wireless communications networks.

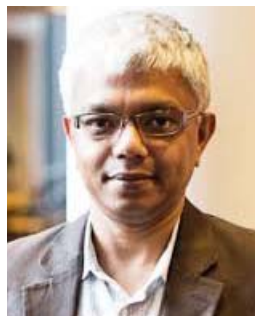

Subhrakanti Dey (SM'06) received the Bachelor in Technology and Master in Technology degrees from the Department of Electronics and Electrical Communication Engineering, IIT Kharagpur, Kharagpur, in 1991 and 1993, respectively, and the Ph.D. degree from the Department of Systems Engineering, Research School of Information Sciences and Engineering, Australian National University, Canberra, in 1996.

Prior to this, he was a Professor with the Department of Electrical and Electronic Engineering, The University of Melbourne, Parkville, Australia, from 2000 until early 2013, and a Professor of telecommunications with the University of South Australia from 2017 to 2018. From September 1995 to September 1997 and September 1998 to February 2000, he was a Post-Doctoral Research Fellow with the Department of Systems Engineering, Australian National University. From September 1997 to September 1998, he was a Post-Doctoral Research Associate with the Institute for Systems Research, University of Maryland, College Park. He is currently a Professor with the Hamilton Institute, National University of Ireland Maynooth, Maynooth, Ireland. He is also a Professor with the Department of Engineering Sciences, Uppsala University, Sweden. His current research interests include wireless communications and networks, signal processing for sensor networks, networked control systems, and molecular communication systems.

Prof. Dey currently serves on the Editorial Board for IEEE CONTROL Systems LetTers, IEEE TRANSACTIONS ON CONTROL OF NETWORK SySTEMS, and IEEE TRANSACTIONS ON WIRELESS COMMUNICATIONS $\mathrm{He}$ was also an Associate Editor of IEEE TRANSACTIONS ON SIGNAL PRocessing from 2007 to 2010 and from 2014 to 2018, IEEE TRANSACTIONS On Automatic Control from 2004 to 2007, and Systems and Control Letters (Elsevier) from 2003 to 2013. 